\title{
H-minimal graphs of low regularity in $\mathbb{H}^{1}$
}

\author{
Scott D. Pauls*
}

\begin{abstract}
In this paper we investigate H-minimal graphs of lower regularity. We show that noncharacteristic $C^{1} \mathrm{H}$-minimal graphs whose components of the unit horizontal Gauss map are in $W^{1,1}$ are ruled surfaces with $C^{2}$ seed curves. Moreover, in light of a structure theorem of Franchi, Serapioni and Serra Cassano, we see that any H-minimal graph is, up to a set of perimeter zero, composed of such pieces. Along these lines, we investigate ways in which patches of $C^{1}$ $\mathrm{H}$-minimal graphs can be glued together to form continuous piecewise $C^{1} \mathrm{H}$-minimal surfaces.

We apply this description of H-minimal graphs to the question of the existence of smooth solutions to the Dirichlet problem with smooth data. We find a necessary and sufficient condition for the existence of smooth solutions and produce examples where the conditions are satisfied and where they fail. In particular we illustrate the failure of the smoothness of the data to force smoothness of the solution to the Dirichlet problem by producing a class of curves whose H-minimal spanning graphs cannot be $C^{2}$.
\end{abstract}

Mathematics Subject Classification (2000). 53C17, 53A10.

Keywords. Carnot-Carathéodory geometry, minimal surfaces, Plateau problem.

\section{Introduction}

In this paper, we further investigate the properties of $\mathrm{H}$-minimal surfaces in the Heisenberg group with a focus on the regularity of H-minimal surfaces that satisfy Dirichlet boundary conditions.

The study of H-minimal surfaces was introduced in the foundational paper of Garofalo and Nhieu ([11]) where they showed the existence of H-minimal surfaces of bounded variation that satisfy certain boundary conditions. Expanding on these results, several authors extended the investigation showing different properties and constructions of H-minimal surfaces in various settings (see, for example, [1], [4], [5], [6], [7], [12], [14], [15], [16], [17]). Recently, N. Garofalo and the author ([12]) gave a characterization of $C^{2} \mathrm{H}$-minimal surfaces used to investigate an analogue of the Bernstein problem in the Heisenberg group. A different approach to the study

\footnotetext{
${ }^{*}$ The author is partially supported by NSF grant DMS-0306752
} 
of analogues of the Bernstein problem was completed by Cheng, Hwang, Malchiodi and Yang in [5] and [4]. We note that there is some overlap between the results in [12] and those of [5] and [4] but that the techniques are independent. In particular, both [12] and [5] make the observation that $C^{2} \mathrm{H}$-minimal surfaces are ruled surfaces but analyze them using different tools (in fact, [5] uses the machinery of pseudohermitian geometry and hence many of their results apply to a larger class of Carnot-Carathéodory spaces). Using the machinery of [5], two of the authors classify properly embedded H-minimal surfaces in the Heisenberg group in [4] while [12] gives a geometric description of the properties of embedded H-minimal surfaces which are graphs over some plane. Again, the results overlap in some respects, but the techniques are independent.

With respect to the discussion in this paper, we will use the tools developed in [12]. For the purposes of this paper, the two most important theorems from [12] are:

Theorem 1.1. Let $k \geq 2$. A noncharacteristic patch of a $C^{k}$ surface $S \subset \mathbb{H}^{1}$ of the type

$$
S=\left\{(x, y, t) \in \mathbb{H}^{1} \mid(x, y) \in \Omega, t=h(x, y)\right\},
$$

where $h: \Omega \rightarrow \mathbb{R}$ is a $C^{k}$ function over an open domain $\Omega$ in the $x y$-plane, is an $H$-minimal surface if and only if for every $p \in S$, there exists a neighborhood $U$ of $p$ so that $U$ can be parameterized by

$$
(s, r) \rightarrow\left(\gamma_{1}(s)+r \gamma_{2}^{\prime}(s), \gamma_{2}(s)-r \gamma_{1}^{\prime}(s), h(s, r)\right),
$$

where

$$
h(s, r)=h_{0}(s)-\frac{r}{2}\left\langle\gamma(s), \gamma^{\prime}(s)\right\rangle
$$

and

$$
\gamma \in C^{k+1}, \quad h_{0} \in C^{k}
$$

Thus, to specify such a patch of smooth H-minimal surface, one must specify a single curve in $\mathbb{H}^{1}$ determined by a curve in the plane, $\gamma(s)$, parameterized by arc-length, and an initial height function $h_{0}(s)$.

The curve $\gamma(s)$ in the theorem is called a seed curve and determines almost all of the behavior of the neighborhood $U$. Indeed, under the assumption of at least $C^{2}$ smoothness, we have:

Theorem 1.2. Let $S \subset \mathbb{H}^{1}$ be a $C^{2}$ connected, open, complete and embedded $H$ minimal surface. Then, either $S$ is a vertical plane, or $S$ is determined by a generalized seed curve $\Gamma=\left\{\left(\gamma_{1}^{i}(s), \gamma_{2}^{i}(s), h_{0}^{i}(s)\right)\right\}$.

A generalized seed curve is a collection of seed curves, height functions and patching data which, taken together, give a description of a single curve in $\mathbb{H}^{1}$. In other words, for such H-minimal surfaces, a single curve determines the entire surface. 
As all H-minimal surfaces have locally finite perimeter (i.e. they are X-Caccioppoli sets), we turn now to the work of Franchi, Serra Cassano and Serapioni ([10]) and recall the following theorem.

Theorem 1.3. Let $E \subset \mathbb{H}^{1}$ be an $X$-Caccioppoli set, then the reduced boundary of $E, \partial_{X}^{*} E$, is $X$-rectifiable, i.e.,

$$
\partial_{X}^{*} E=N \cup \bigcup_{j=1}^{\infty} K_{j},
$$

where $\mathscr{H}_{\mathrm{CC}}^{3}(N)=0$, and $K_{j}$ is a compact subset of a non-characteristic hypersurface $S_{j}$ of class $C_{\mathbb{H}}^{1}$. Moreover, one has for any $g \in K_{j}$ and every $\xi \in T_{H, g} S_{j}$

$$
\left\langle v_{X}^{E}(g), \xi\right\rangle=0,
$$

where $v_{X}^{E}(g)$ denotes the generalized horizontal outer normal to $E$ in $g, T_{H, g} S_{j}$ indicates the non-characteristic plane orthogonal to the horizontal normal to $S_{j}$ in $g, \mathscr{H}_{\mathrm{CC}}^{3}$ is the 3-dimensional Hausdorff measure in $\mathbb{H}^{1}$ constructed with respect to the Carnot-Carathéodory distance and $C_{\mathbb{H}}^{1}$ is the space of functions which are horizontally continuously differentiable, i.e. $X_{1} f, X_{2} f$ exist and are continuous.

The reduced boundary, $\partial_{X}^{*}$, is the set of boundary points where the unit horizontal Gauss map is well-defined (see the next section for a precise definition). For the discussion of this paper, it is important to note that the reduced boundary is a full measure subset of the boundary. The main point of this theorem is that H-minimal surfaces can be decomposed into a set of $\mathscr{H}_{\mathrm{CC}}^{3}$-measure zero and a union of $C_{\mathbb{H}}^{1}$ sets. As we will restrict ourselves to investigating graphs over the $x y$-plane, we remind the reader that a $C_{\mathbb{H}}^{1}$ graph is $C^{1}$. This, of course, leaves a gap - the pieces given by Theorem 1.3 are $C^{1}$ while Theorem 1.2 applies only to $C^{2}$ surfaces. The first goal of this paper is to partially bridge the gap between the two theorems.

Theorem A. If $S$ is an open $C^{1} H$-minimal graph over a domain $\Omega \subset \mathbb{R}^{2}$ with no characteristic points and unit horizontal Gauss map $\boldsymbol{v}_{X}$ whose components are in $W^{1,1}(\Omega)$, then the integral curves of $\boldsymbol{v}_{X}{ }^{\perp}$ are straight lines and $S$ can be locally parameterized by

$$
(s, r) \rightarrow\left(\gamma_{1}(s)+r \gamma_{2}^{\prime}(s), \gamma_{2}(s)-r \gamma_{1}^{\prime}(s), h(s, r)\right),
$$

where

$$
h(s, r)=h_{0}(s)-\frac{r}{2}\left\langle\gamma(s), \gamma^{\prime}(s)\right\rangle
$$

and $\gamma$ is an integral curve of $\boldsymbol{v}_{X}$. Moreover, if there exists $\varepsilon>0$ so that $d(\Omega, \gamma)>$ $\varepsilon>0$ then $h_{0}(s) \in C^{1}$ and $\gamma(s) \in C^{2}$. 
In this theorem $d(\Omega, \gamma)$ is a measure of the "horizontal thickness" of the set $\Omega$ (see Definition 3.5 for a precise statement).

This theorem is shown in a series of steps. First we show that the weak directional derivative of $\boldsymbol{v}_{X}$ in the direction of $\boldsymbol{v}_{X}{ }^{\perp}$ is zero. This is enough to show that the integral curves of $\boldsymbol{v}_{X}{ }^{\perp}$ are lines. Second, forming $\gamma(s)$ as the integral curve of $\boldsymbol{v}_{X}$, a geometric argument shows that $\gamma^{\prime}(s)$ is Lipschitz. Coupled with a further estimate, this shows that $\gamma^{\prime \prime}(s)$ exists and is continuous. Applying arguments similar to those in [12] yields the representation given in the theorem.

Combining this theorem with Theorem 1.3 of Franchi, Serapioni and Serra Cassano yields:

Theorem B. If $S$ is an H-minimal graph then

$$
S=N \cup \bigcup_{i=1}^{\infty} K_{i},
$$

where $N$ is a set of $\mathscr{H}_{\mathrm{CC}}^{3}$-measure zero and each $K_{i}$, a graph over $\Omega_{i}$, with its unit horizontal Gauss map in $W^{1,1}(\Omega)$ and $d\left(K_{i}, \gamma\right)>\varepsilon>0$ is a compact piece of a $C^{1}$ $H$-minimal graph which can locally be parameterized by equations (3) and (4) with $\gamma \in C^{2}$ and $h_{0} \in C^{1}$.

While this theorem recovers the characterization of $\mathrm{H}$-minimal surfaces as ruled surfaces in the Heisenberg group, it still leaves a gap between the baseline results of Franchi, Serra Cassano and Serapioni and Theorem 1.1. Specifically, Theorem 1.3 allows that the $C^{1}$ pieces may be glued together in nonsmooth ways. We find that this can happen:

Theorem C. Suppose $S_{1}$ and $S_{2}$ are subsets of $C^{1} H$-minimal graphs with no characteristic points, each parameterized by a single seed curve and height function, defined over closed sets $\Omega_{1}, \Omega_{2} \subset \mathbb{R}^{2}$ with open interior, $C=\Omega_{1} \cap \Omega_{2}$ a $C^{1}$ curve, $\partial \Omega_{i} \in C^{1}$ and $d\left(\Omega_{i}\right)>0$ for $i=1,2$. Moreover, let $v_{1}=\left(\bar{p}_{1}, \bar{q}_{1}\right)$ and $v_{2}=\left(\bar{p}_{2}, \bar{q}_{2}\right)$ be the respective unit horizontal Gauss maps. Then $S_{1} \cup S_{2}$ is an H-minimal graph if and only if $\left.\left(v_{1}-v_{2}\right)\right|_{C}$ is tangent to $C$ almost everywhere.

This provides one way in which a continuous, piecewise $C^{1} \mathrm{H}$-minimal graph is constructed.

This brings forward an obvious question:

In standard minimal surface theory, the solutions to the minimal surface equation subject to Dirichlet boundary conditions gain additional regularity from the regularity of the boundary. Do H-minimal surfaces have a similar property? 
We devote the remaining part of the paper to exploring this question. First, we examine some of the best behaved H-minimal surfaces, those that are minimal in Riemannian approximators of $\mathbb{H}^{1}$ as well as H-minimal. We call these persistent minimal surfaces and classify them.

Theorem D. The persistent H-minimal graphs fall into two categories:

(1) $S$ is given by $(x, y, u(x, y))$ where

$$
\begin{aligned}
u(x, y)= & \frac{m}{1+m^{2}}\left(x-x_{0}\right)^{2}+\frac{m^{2}-1}{m^{2}+1}\left(x-x_{0}\right)\left(y-y_{0}\right)-\frac{m}{1+m^{2}}\left(y-y_{0}\right)^{2} \\
& +\frac{a}{\sqrt{1+m^{2}}}\left(x-x_{0}\right)+\frac{a m}{\sqrt{1+m^{2}}}\left(y-y_{0}\right)+b
\end{aligned}
$$

for $m, a, b, x_{0}, y_{0} \in \mathbb{R}$.

(2) S, given in cylindrical coordinates, is

$$
(\rho \cos (\theta), \rho \sin (\theta), a \theta+b)
$$

for $a, b \in \mathbb{R}$.

These surfaces give examples of the best possible case - they are $C^{\infty}$ spanning surfaces. Second, in Section 7, we consider the question of the existence of smooth minimal spanning surfaces. For a fixed smooth closed curve, we focus on finding the $C^{1}$ smooth ruled $\mathrm{H}$-minimal spanning graphs, those $C^{1} \mathrm{H}$-minimal graphs which satisfy the additional condition that the rules may be extended over the characteristic locus (as straight lines). We note that by work in [12] or [5], all $C^{2} \mathrm{H}$-minimal graphs satisfy this condition. However, using techniques similar to those of Theorem C, one can construct $C^{1}$ minimal graphs that are the union of two ruled surfaces along a common characteristic locus and do not satisfy this condition. For simplicity, we will ignore this type of construction. Taking the characterization of H-minimal surfaces as ruled surfaces, we create a necessary and sufficient condition for a smooth closed curve which is the graph over a curve in the $x y$-plane to be spanned by a $C^{1}$ ruled $\mathrm{H}$-minimal graph.

Existence Criteria. Given a closed smooth curve $c(\theta)=\left(c_{1}(\theta), c_{2}(\theta), c_{3}(\theta)\right)$ which is a graph over a curve in the $x y$-plane, $c$ is spanned by a $C^{1}$ ruled $H$-minimal graph if and only if there exists a monotone $C^{1}$ function $\varphi: S^{1} \rightarrow S^{1}$ with $\varphi(\theta) \in A(\theta)$.

In this statement, $A(\theta)$ is the set of points on $c$ that are accessible from $c(\theta)$ via a rule of an $\mathrm{H}$-minimal surface:

$$
A(\theta)=\left\{\theta_{0} \mid c_{3}\left(\theta_{0}\right)-c_{3}(\theta)-\frac{1}{2} c_{1}\left(\theta_{0}\right) c_{2}(\theta)+\frac{1}{2} c_{1}(\theta) c_{2}\left(\theta_{0}\right)=0\right\} .
$$


The examples in this section show curves that satisfy the criteria and curves that exhibit an obstruction. We also discuss the genericity of these classes. Finally, we show that there are many curves, $c$, which do not have smooth ruled H-minimal spanning graphs. This provides an upper bound on the regularity of the solution to the Plateau Problem for these curves: the solution to the Plateau Problem cannot be a $C^{1}$ ruled minimal graph.

Theorem E. Suppose $c$ is a $C^{1}$ curve with no Legendrian points which is spanned by a $C^{1}$ smooth ruled $H$-minimal graph, $S$. Then there exists an interval, I, so that $c(I)$ is contained in a plane.

Corollary 1.4. If $c$ is a smooth curve with no Legendrian points and no portion of $c$ is contained in a plane then an $\mathrm{H}$-minimal surface spanning c cannot be a $\mathrm{C}^{1}$ ruled H-minimal surface.

These different examples show that solutions to the Dirichlet problem and the Plateau Problem may not have any specified regularity. In particular, the persistent $\mathrm{H}$-minimal graphs show that some curves have a $C^{\infty}$ solution to the Plateau Problem while the subsequent examples show instances where $C^{\infty}$ curves may not have a solution to the Dirichlet problem of high regularity. Indeed, the last set of examples show that for certain totally non-Legendrian curves, the graphical solutions to the Dirichlet (and hence the Plateau) problem are necessarily at most $C^{1}$ but cannot be ruled surfaces. A consequences of this is that these surfaces must have unresolvable discontinuities in their unit horizontal Gauss maps. We reiterate that Theorem B shows that any H-minimal graph is piecewise $C^{1}$ and its seed curve, on the $C^{1}$ patches, is $C^{2}$.

The author would like to thank the referee for many helpful comments and suggestions. The author would also like to thank J. H. Cheng, J. F. Hwang and P. Yang for pointing out an error in an earlier version of this paper.

\section{Definitions and notation}

Throughout this paper, we restrict our attention to the topologically three dimensional Heisenberg group, $\mathbb{H}^{1}$. For convenience, we represent $\mathbb{H}^{1}$ via an identification with $\mathbb{R}^{3}$. Considering $\mathbb{R}^{3}$ with its usual coordinates labeled as $\{x, y, t\}$, we define the following vector fields:

$$
X_{1}=\frac{\partial}{\partial x}-\frac{y}{2} \frac{\partial}{\partial t}, \quad X_{2}=\frac{\partial}{\partial y}+\frac{x}{2} \frac{\partial}{\partial t}, \quad T=\frac{\partial}{\partial t} .
$$

The vector fields $\left\{X_{1}, X_{2}, T\right\}$ form a basis for the Lie algebra of $\mathbb{H}^{1}$ at any point $(x, y, t)$. Note that, via the exponential map at the origin, we identify $\mathbb{H}^{1}$ with $\mathbb{R}^{3}$ 
using these coordinates, denoting the point $e^{\alpha X_{1}+\beta X_{2}+\gamma T}$ by $(\alpha, \beta, \gamma)$. For the purposes of this paper, we define a left invariant inner product on $\mathbb{H}^{1},\langle\cdot, \cdot\rangle$, which makes $\left\{X_{1}, X_{2}, T\right\}$ an orthonormal basis at each point. Notice that at each point, $\left[X_{1}, X_{2}\right]=T$ and hence $\left\{X_{1}, X_{2}\right\}$ is a bracket generating set for $\mathbb{H}^{1}$. We define a subbundle on $\mathbb{H}^{1}$, called the horizontal subbundle of $\mathbb{H}^{1}$, by

$$
H \mathbb{H}^{1}=\left\{(x, y, t, w) \in \mathbb{H}^{1} \times \mathbb{R}^{3} \mid w \in \operatorname{span}\left\{X_{1}, X_{2}\right\}\right\} .
$$

The single nontrivial bracket relation yields the following multiplication law via the Campbell-Baker-Hausdorff formula:

$$
(a, b, c)(\alpha, \beta, \gamma)=\left(a+\alpha, b+\beta, c+\gamma+\frac{1}{2}(a \beta-\alpha b)\right)
$$

To define the Carnot-Carathéodory metric on $\mathbb{H}^{1}$, we construct a path metric. Letting $\mathcal{A}(m, n)$ be the set of all absolutely continuous paths in $\mathbb{H}^{1}$ so that $\gamma(0)=m$, $\gamma(1)=n$ and $\gamma^{\prime}(t) \in H_{\gamma(t)} \mathbb{H}^{1}$ when $\gamma^{\prime}(t)$ exists we define:

$$
d_{\mathrm{CC}}(m, n)=\inf _{\gamma \in \mathcal{A}(m, n)}\left\{\int_{I}\left\langle\gamma^{\prime}(t), \gamma^{\prime}(t)\right\rangle^{\frac{1}{2}}\right\} .
$$

Note that, since $\langle\cdot, \cdot\rangle$ is left invariant, so is $d_{\mathrm{CC}}$. Moreover, $d_{\mathrm{CC}}$ admits a homothety at each point $(x, y, t)$ :

$$
h_{s}(x, y, t)=\left(s x, s y, s^{2} t\right)
$$

whereby

$$
d_{\mathrm{CC}}\left(h_{s} m, h_{s} n\right)=s d_{\mathrm{CC}}(m, n) .
$$

We denote by $\mathscr{H}_{\mathrm{CC}}^{k}$ the $k$-dimensional spherical Hausdorff measure constructed from $d_{\mathrm{CC}}$.

Definition 2.1. The horizontal gradient operator is

$$
\nabla_{0}=\left(X_{1}, X_{2}\right)
$$

Hence,

$$
\nabla_{0} f=\left(X_{1} f\right) X_{1}+\left(X_{2} f\right) X_{2} .
$$

The horizontal divergence of a vector field $V=v_{1} X_{1}+v_{2} X_{2}$ is

$$
\operatorname{div}_{0} V=\nabla_{0} \cdot V=X_{1} v_{1}+X_{2} v_{2} .
$$

In addition to the three dimensional Hausdorff measure, we recall the perimeter measure introduced independently by Capogna, Danielli and Garofalo ([2], [3]) and Franchi, Gallot and Wheeden ([9]). 
Definition 2.2. Let $\Omega$ be an open subset of $\mathbb{H}^{1}$. We say that $f: \Omega \rightarrow \mathbb{R}$ is of bounded variation (i.e. $f \in \mathrm{BV}_{\mathbb{H}^{1}}(\Omega)$ ) if

- $f \in L^{1}(\Omega)$

- $\sup \left\{\int f \operatorname{div}_{0} V d h\left|V \in C_{0}^{1}\left(\Omega, H \mathbb{H}^{1}\right),\right| V \mid \leq 1\right\}<\infty$.

We define $\mathrm{BV}_{\mathbb{H}^{1}, \text { loc }}(\Omega)$ analogously.

Definition 2.3. We say that $E \subset \mathbb{H}^{1}$ is an $X$-Caccioppoli set if the characteristic function $\chi_{E}$ of $E$ is contained in $\mathrm{BV}_{\mathbb{H}^{1}}$, loc . The measure $\left|\nabla_{0} \chi_{E}\right|$ is called the perimeter measure and will be denoted by $\mathcal{P}$.

We recall that (see [11], [10]) if $\partial E$ is a smooth surface given by $t=u(x, y)$, then $\mathcal{P}$ is mutually absolutely continuous with $\mathcal{H}_{\mathrm{CC}}^{3}$. Moreover, up to a choice of constant, $\mathcal{H}_{\mathrm{CC}}^{3}(\partial E)$ is given by

$$
\int_{\partial E} \sqrt{\left(X_{1}(t-u(x, y))\right)^{2}+\left(X_{2}(t-u(x, y))\right)^{2}} d A .
$$

Again from [10], we recall the definition of the generalized horizontal normal.

Definition 2.4. There exists a $\mathcal{P}$ measurable section $v_{E}$ of $H \mathbb{H}^{1}$ such that

$$
-\int_{E} \operatorname{div}_{0} \varphi d h=\int_{\mathbb{H}^{1}}\left\langle v_{E}, \varphi\right\rangle d \mathcal{P}
$$

for all $\varphi \in C_{0}^{\infty}\left(\Omega, H \mathbb{H}^{1}\right),\left|v_{E}(p)\right|=1$ for $\mathcal{P}$ a.e. $p \in \mathbb{H}^{1}$.

We next recall the definition of the reduced boundary:

Definition 2.5. Let $E$ be an X-Caccioppoli set. Let $U(p, r)$ be the open ball of radius $r$ and center $p$. A point $p$ is in the reduced boundary of $E, p \in \partial_{\mathbb{H}^{1}}^{*} E$, if

(1) $\mathcal{P}(U(p, r))>0$ for any $r>0$,

(2) $\lim _{r \rightarrow 0} \frac{1}{\mathcal{P}(U(p, r))} \int_{U(p, r)} v_{E} d \mathcal{P}$ exists,

(3) $\left|\lim _{r \rightarrow 0} \frac{1}{\mathcal{P}(U(p, r))} \int_{U(p, r)} v_{E} d \mathcal{P}\right|=1$.

We note that Lemma 7.3 in [10] ensures that $\partial_{\mathbb{H}^{1}}^{*} E$ has full $\mathcal{P}$ measure in $\partial E$.

In this paper, we will be examining smooth graphs over the $x y$-plane in $\mathbb{H}^{1}$ by which we mean surfaces which can be represented as $t=u(x, y)$ using the coordinates described above. As shown in [16] and [7], H-minimal surfaces are critical points of an area functional based on the horizontal Gauss map of the surface 
$t=u(x, y)$. The horizontal Gauss map is the projection of the Riemannian normal of the surface to the horizontal bundle:

$$
\begin{aligned}
G: S & \rightarrow H S \\
G(x, y, u(x, y)) & =\left(X_{1}(t-u(x, y))\right) X_{1}+\left(X_{2}(t-u(x, y))\right) X_{2} .
\end{aligned}
$$

We give classically inspired names to these horizontal derivatives of $f$, letting

$$
\begin{aligned}
& p=X_{1}(t-u(x, y)), \\
& q=X_{2}(t-u(x, y)) .
\end{aligned}
$$

In this paper the unit horizontal Gauss map plays a crucial role and so we define the unit horizontal Gauss map by

$$
v_{X}=\bar{p} X_{1}+\bar{q} X_{2}
$$

where $\bar{p}=\frac{p}{\sqrt{p^{2}+q^{2}}}$ and $\bar{q}=\frac{q}{\sqrt{p^{2}+q^{2}}}$. Notice that $\boldsymbol{v}_{X}$ has a limited domain and is not defined at points where both $p$ and $q$ are zero. Such points are called characteristic points and play an important role in the study of surfaces in Carnot-Carathéodory spaces.

In this paper we consider surfaces which are graphs over the $x y$-plane. In other words, the set $E$ in the previous theorem is given as

$$
\{(x, y, t) \mid t<u(x, y)\} .
$$

Thus the hypersurface $\partial E$ would be given as $t-u(x, y)=0$. The function $t-$ $u(x, y)$ is horizontally continuously differentiable if and only if $u$ is continuously differentiable.

With this notation in place, we next review the characterization of smooth noncharacteristic area minimizing graphs by an appropriate partial differential equation via the first variation of the energy. The first variation formula has been explored in a variety of settings by a number of authors including the aforementioned paper of Cheng, Hwang, Malchiodi and Yang ([5]), Danielli, Garofalo and Nhieu ([7]), the author ([16]), Bonk and Capogna ([1]), Ritoré and Rosales ([17]). For the convenience of the reader, we recall the derivation of the equation here. First, the energy integral we use for the variational setup is

$$
E(u)=\int_{\Omega} \sqrt{p^{2}+q^{2}},
$$

where $t=u(x, y)$ defines the graph in question over a domain $\Omega$ and has at least two weak derivatives.

Second, we consider a variation in the $t$ direction by a function $\varphi(x, y) \in C_{0}^{\infty}(\Omega)$. Then,

$$
E(\varepsilon)=E(u+\varepsilon \varphi)=\int_{\Omega} \sqrt{\left(u_{x}+\frac{y}{2}+\varepsilon \varphi_{x}\right)^{2}+\left(u_{y}-\frac{x}{2}+\varepsilon \varphi_{y}\right)^{2}} .
$$


Abusing notation, we let $p=u_{x}+\frac{y}{2}$ and $q=u_{y}-\frac{x}{2}$. Thus, differentiating with respect to $\varepsilon$ twice and evaluating at zero, we have

$$
E^{\prime}(0)=\int_{\Omega} \frac{p \varphi_{x}+q \varphi_{y}}{\sqrt{p^{2}+q^{2}}}
$$

and

$$
E^{\prime \prime}(0)=\int_{\Omega} \frac{\left(q \varphi_{x}-p \varphi_{y}\right)^{2}}{\left(p^{2}+q^{2}\right)^{\frac{3}{2}}}=\int_{\Omega} \frac{\left(\nabla \varphi \cdot G^{\perp}\right)^{2}}{|G|^{3}} .
$$

In the last equation we use the convention that if $v$ is the vector given by coordinates $(a, b)$ then $v^{\perp}$ is given by $(b,-a)$. This convention will be used throughout the paper. Note that the integrand of the second integral is nonnegative and is strictly positive if $\nabla \varphi$ is not parallel to the vector $G$. Thus, to check if a given solution to the Euler-Lagrange equation is a local minimum of area (with respect to this type of perturbation), one must only check it against variations in this direction.

Lemma 2.6. Let $u: \Omega \rightarrow \mathbb{R}, u \in C^{2}$, be a critical point of the energy functional. Then, for all test functions $\varphi \in C_{0}^{\infty}(\Omega), E^{\prime \prime}(0)>0$.

Proof. Notice first that if $\nabla \varphi \cdot G^{\perp}$ is not identically zero on a set of positive measure, then since the integrand is always positive, the result follows. If $\nabla \varphi$ points in the same direction as $G$, we now verify that for such a perturbation, $E^{\prime \prime}>0$. In this case let $\beta$ be a function so that $\nabla \varphi=\beta(x, y) G=\left(\beta G_{1}, \beta G_{2}\right)$. Then $\beta G$ is the gradient of the $C_{0}^{\infty}(\Omega)$ function $\varphi$, so

$$
\varphi_{x y}-\varphi_{y x}=\beta_{y} G_{1}+\beta G_{1, y}-\beta_{x} G_{2}-\beta G_{2, x}=0 .
$$

But

$$
\beta G_{1, y}-\beta G_{2, x}=\beta\left(u_{x y}-u_{y x}-1\right)=-\beta
$$

So we have

$$
-\nabla \beta \cdot G^{\perp}-\beta=0 .
$$

By Theorem 1.1 in the introduction, we see that the integral curves of $G^{\perp}$ are straight lines (for a more detailed discussion of this fact, see [12]). Thus, (5) implies that when $\beta$ is restricted to such a straight line, we have $\beta^{\prime}=-\beta$ and hence $\beta=C e^{-t}$, where $t$ is the parameter along the integral curve. However, as $\varphi$ is compactly supported in $\Omega, \beta$ must tend to zero towards the boundary of $\Omega$. This is a contradiction of the existence of a compactly supported normal variation $\varphi$ with gradient pointing in the same direction as $G$.

Thus local minima of this area functional appear as solutions of the following partial differential equation:

$$
X_{1} \bar{p}+X_{2} \bar{q}=0
$$


This equation says simply that the unit horizontal Gauss map is (horizontally) divergence free:

$$
\operatorname{div}_{0} \boldsymbol{v}_{X}=0 .
$$

In this paper we will also allow solutions that are only weak solutions to this equation. In Section 4 we discuss a condition under which a piecewise $C^{1}$ graph can satisfy this equation weakly, but not strongly.

Remark 1. In some of the references given above, there are versions of these first and second variation equations for more generally defined surfaces. For example, in [7], the authors give these formulae for surfaces defined implicitly as $\varphi(x, y, t)=0$. We also point out that without our restriction to graphs (and perturbations that remain graphs) the second variation formula does not necessarily ensure that the critical points are local minima. Again see the examples in [7].

In [7], Danielli, Garofalo and Nhieu introduce the notation of horizontal mean curvature, which is used to define H-minimal surfaces in both [7] and [12]. We recall slight variations of these definitions here using the notation above.

Definition 2.7. The horizontal mean curvature of $S$ at noncharacteristic points of $S$ is defined by

$$
H=X_{1} \bar{p}+X_{2} \bar{q}
$$

If $x_{0} \in \Sigma$, then

$$
H\left(x_{0}\right)=\lim _{x \rightarrow x_{0}, x \notin \Sigma} H(x) .
$$

provided that the limit exists, finite or infinite. If the limit does not exist, the horizontal mean curvature is not defined at such points.

This definition differs from that in [7] by a constant.

In [7] and [12] a $C^{2}$ surface is called an H-minimal surface if $H$ is identically zero. In this paper, we make a slightly different definition,

Definition 2.8. A $C^{1}$ graph $S$ over a domain $\Omega \subset \mathbb{R}^{2}$ is an $H$-minimal surface if, for any subdomain $\Omega^{\prime} \subset \Omega$ over which $S$ is noncharacteristic, it satisfies (6) weakly. More precisely, if $v_{X}=\bar{p} X_{1}+\bar{q} X_{2}$ is the unit horizontal Gauss map of $S$, then

$$
\int_{\Omega^{\prime}} \bar{p} \varphi_{x}+\bar{q} \varphi_{y} d x d y=0
$$

for all $\varphi \in C_{0}^{\infty}\left(\Omega^{\prime}\right)$.

For completeness, we also recall some of the results of [12]. 
Definition 2.9. Let $S$ be a $C^{2}$ H-minimal graph and $\boldsymbol{v}_{X}$ be its unit horizontal Gauss map. Thinking of $\boldsymbol{v}_{X}$ as a vector field on $\mathbb{R}^{2}$, any integral curve of $\boldsymbol{v}_{X}$ is called a seed curve of $S$. We denote a seed curve by $\gamma_{z}(s)$, i.e. $\gamma_{z}(0)=z, \gamma_{z}^{\prime}(s)=\boldsymbol{v}_{X}\left(\gamma_{z}(s)\right)$. If a basepoint is understood, we denote the curve by $\gamma(s)$. We denote the integral curves of $\boldsymbol{v}_{X}{ }^{\perp}$ by $\mathcal{L}_{Z}(r)$ (or, simply $\mathcal{L}(r)$ if a basepoint is understood).

As mentioned in the introduction, in [12], N. Garofalo and the author show that, for $C^{2} \mathrm{H}$-minimal surfaces, $\mathcal{L}_{z}(r)$ are straight lines in the plane and lift to horizontal lines in $\mathbb{H}^{1}$. This yields an adapted parameterization of the plane:

$$
F(s, r)=\left(\gamma_{1}(s)+r \gamma_{2}^{\prime}(s), \gamma_{2}-r \gamma_{1}^{\prime}(s)\right) .
$$

We recall that this parameterization ceases to be a local diffeomorphism along the locus $r=\frac{1}{\kappa(s)}$ where $\kappa$ is the signed curvature of the seed curve $\gamma$ and is given by

$$
\kappa(s)=\gamma^{\prime \prime}(s) \cdot \gamma^{\prime}(s)^{\perp} .
$$

When lifted to $\mathbb{H}^{1}$, this yields a parameterization of the H-minimal surface as a ruled surface

$$
S=\left(\gamma_{1}(s)+r \gamma_{2}^{\prime}(s), \gamma_{2}-r \gamma_{1}^{\prime}(s), h_{0}(s)-\frac{r}{2} \gamma \cdot \gamma^{\prime}(s)\right) .
$$

This is the content of Theorem 1.1 in the introduction. Moreover, we can extend this parameterization from this patch of surface to include all of the rules (i.e. allow $r \in(-\infty, \infty))$, which introduces characteristic points at the locus given by

$$
\Sigma(s, r)=h_{0}^{\prime}(s)-r+\frac{1}{2} \gamma^{\prime}(s) \cdot \gamma(s)^{\perp}+\frac{r^{2}}{2} \kappa(s)=0 .
$$

We recall that generically this yields two branches of the characteristic locus, one on one side of the locus $r=\frac{1}{\kappa(s)}$ and the second on the other side of this locus. We refer the reader to [12], Section 7 for a more detailed discussion of these features.

\section{Noncharacteristic $C^{1}$ H-minimal graphs}

In this section we investigate $C^{1} \mathrm{H}$-minimal graphs. We will focus first on section of $C^{1} \mathrm{H}$-minimal graphs that do not have characteristic points. In this setting, we show that such graphs are ruled surfaces as in the $C^{2}$ case. At the end of the section we will address the question of characteristic points. Throughout the section we will consider a surface defined by $(x, y, u(x, y))$ where $u: \Omega \rightarrow \mathbb{R}$ is a $C^{1}$ function. 
3.1. Weak directional derivatives. At first we will assume that the function $u$ defining the H-minimal surface is at least $C^{2}$ and so the components of the unit horizontal Gauss map are continuously differentiable. Under this assumption, we compute the directional derivative of $\bar{p}$ in the direction of $v=\left(1,-\frac{\bar{p}}{\bar{q}}\right)$ (the choice of this vector will become evident in a moment):

$$
D_{v} \bar{p}=\nabla \bar{p} \cdot\left(1,-\frac{\bar{p}}{\bar{q}}\right)=\bar{p}_{x}-\frac{\bar{p}}{\bar{q}} \bar{p}_{y}=\bar{p}_{x}+\bar{q}_{y} .
$$

The last equation is true because $\bar{q}=\sqrt{1-\bar{p}^{2}}$ and hence $\bar{q}_{y}=-\frac{\bar{p}}{\bar{q}} \bar{p}_{y}$.

Thus we can interpret the integral equation

$$
-\int_{\Omega} \bar{p} \varphi_{x}+\bar{q} \varphi_{y} d x d y=0
$$

as a weak form of the equation

$$
\nabla \bar{p} \cdot\left(1,-\frac{\bar{p}}{\bar{q}}\right)=0
$$

In other words, if $S$ is an $\mathrm{H}$-minimal surface, then $\bar{p}$ is weakly constant in the $\boldsymbol{v}_{X}{ }^{\perp}$ direction $\left(\boldsymbol{v}_{X}{ }^{\perp}\right.$ and $\left(1,-\frac{\bar{p}}{\bar{q}}\right)$ point in the same direction). We take this as a definition.

Definition 3.1. The directional derivative of $\bar{p}$ in the direction of $v=\left(1,-\frac{\bar{p}}{\bar{q}}\right)$ is weakly zero if

$$
-\int_{\Omega} \bar{p} \varphi_{x}+\bar{q} \varphi_{y} d x d y=0 .
$$

In this case we write $D_{v} \bar{p}=0$

3.2. Rulings of $\boldsymbol{C}^{\mathbf{1}} \mathbf{H}$-minimal graphs. Mimicking classical arguments we have the following result.

Lemma 3.2. Let $v=\left(1,-\frac{\bar{p}}{\bar{q}}\right)$ where $(\bar{p}, \bar{q})$ is the unit horizontal Gauss map of an $H$-minimal graph, $S$, over a domain $O \subset \mathbb{R}^{2}$ and $\bar{p}, \bar{q} \in W^{1,1}(O)$. Assume $\Omega \subset O$ is an open domain so that

(1) The portion of $S$ over $\Omega$ is noncharacteristic.

(2) $v$ is continuous on $\Omega$ (i.e. $\bar{q} \neq 0$ ).

Finally, let $c_{x}(t)$ be an integral curve of $v$ with $c_{x}(0)=x$ and let $D_{v}^{h} \bar{p}(x)=$ $\frac{\bar{p}\left(c_{x}(h)\right)-\bar{p}(x)}{h}$. Then for $V$ compactly contained in $\Omega, h<\operatorname{dist}(x, \partial \Omega), D_{v}^{h} \bar{p}(x)=0$ for a.e. $x \in V$. 
Proof. Let $w$ be a continuous vector field on $V$ and let $c_{x_{0}}^{w}(h)$ be an integral curve of $w$ passing through the point $x_{0}$. We note that $c_{x_{0}}^{w}(h)$, as a point set, coincides with $\mathcal{L}_{x_{0}}(r)$ but is parametrized differently. We may reparametrize $c_{x_{0}}^{w}$ so that $\left(c_{x_{0}}^{w}\right)^{\prime}(s)=h w$. Assuming briefly that $f$ is a smooth function, we have

$$
\begin{aligned}
f\left(c_{x_{0}}^{w}(h)\right)-f\left(c_{x_{0}}^{w}(0)\right) & =\int_{0}^{1} \nabla f\left(c_{x_{0}}^{w}(s)\right) \cdot\left(c_{x_{0}}^{w}\right)^{\prime}(s) d s \\
& =\int_{0}^{1} \nabla f\left(c_{x_{0}}^{w}(s)\right) \cdot h w d s \\
& =h \int_{0}^{1} D_{w} f\left(c_{x_{0}}^{w}\right)(s) d s .
\end{aligned}
$$

So,

$$
D_{w}^{h} f=\frac{f\left(c_{x_{0}}^{w}(h)\right)-f\left(c_{x_{0}}^{w}(0)\right)}{h}=\int_{0}^{1} D_{w} f\left(c_{x_{0}}^{w}(s)\right) d s .
$$

Integrating appropriately, we have, for example, that

$$
\int_{V}\left|D_{w}^{h} f\right| d x \leq \int_{V}\left|D_{w} f\right| d x
$$

Using standard mollification we can smooth the function $\bar{p}$ yielding a $C^{\infty}$ function $\bar{p}_{\varepsilon}$. As we have restricted to a set where $\bar{p}$ is continuous (i.e. there are no characteristic points), we know that $\bar{p}_{\varepsilon} \rightarrow \bar{p}$ uniformly as $\varepsilon \rightarrow 0$. Noting that $\bar{p}^{2}, \bar{q}^{2}, \bar{p} \bar{p}_{y}, \bar{q} \bar{q}_{y} \in$ $L^{1}(\Omega)$, we have that $\left(\bar{p}^{2}+\bar{q}^{2}\right)_{y}=2 \bar{p} \bar{p}_{y}+2 \bar{q} \bar{q}_{y}=0$ in $L^{1}$. Since $\frac{\bar{q}}{\bar{p}}$ is continuous on $\Omega$ we have $-\frac{\bar{q}}{\bar{p}} \bar{y}_{y}=\bar{q}_{y}$ in $L_{\mathrm{loc}}^{1}(\Omega)$. Thus, $D_{v} \bar{p}=\bar{p}_{x}+\bar{q}_{y}$ in $L_{\mathrm{loc}}^{1}(\Omega)$. Thus, by $H$-minimality, for $\varphi \in C_{0}^{\infty}(\Omega)$,

$$
\int_{\Omega} D_{v} \bar{p} \varphi=-\int_{\Omega} \bar{p} \varphi_{x}+\bar{q} \varphi_{y}=0
$$

and we have $D_{v} \bar{p}=0$.

$$
\begin{aligned}
& \tilde{p}_{\varepsilon}=\frac{\bar{p}_{\varepsilon}}{\sqrt{\bar{p}_{\varepsilon}^{2}+\bar{q}_{\varepsilon}^{2}}}, \\
& \tilde{q}_{\varepsilon}=\frac{\bar{q}_{\varepsilon}}{\sqrt{\bar{p}_{\varepsilon}^{2}+\bar{q}_{\varepsilon}^{2}}},
\end{aligned}
$$

and

$$
\tilde{v}=\left(1,-\frac{\tilde{p}_{\varepsilon}}{\tilde{q}_{\varepsilon}}\right) .
$$


As $\bar{p}_{\varepsilon}$ converges to $\bar{p}$ uniformly on $V$, it follows by direct calculation that $\tilde{p}_{\varepsilon}$ converges to $\bar{p}$ uniformly on $V$ as well. Similarly, $\tilde{q}_{\varepsilon}$ converges to $\bar{q}$ uniformly on $V$ and $\tilde{v}$ converges to $v$ uniformly on $V$.

Under this definition we have that

$$
\tilde{p}_{\varepsilon}^{2}+\tilde{q}_{\varepsilon}^{2}=1
$$

Differentiating with respect to $y$ and solving for $\left(\tilde{p}_{\varepsilon}\right)_{y}$ we have

$$
\left(\tilde{p}_{\varepsilon}\right)_{y}=-\frac{\tilde{q}_{\varepsilon}}{\tilde{p}_{\varepsilon}}\left(\tilde{q}_{\varepsilon}\right)_{y}
$$

So

$$
D_{\tilde{v}} \tilde{p}_{\varepsilon}=\left(\tilde{p}_{\varepsilon}\right)_{x}-\left(\tilde{p}_{\varepsilon}\right)_{y} \frac{\tilde{p}_{\varepsilon}}{\tilde{q}_{\varepsilon}}=\left(\tilde{p}_{\varepsilon}\right)_{x}+\left(\tilde{q}_{\varepsilon}\right)_{y} \frac{\tilde{q}_{\varepsilon}}{\tilde{p}_{\varepsilon}} \frac{\tilde{p}_{\varepsilon}}{\tilde{q}_{\varepsilon}}=\left(\tilde{p}_{\varepsilon}\right)_{x}+\left(\tilde{q}_{\varepsilon}\right)_{y} .
$$

As $\left(\bar{p}_{x}\right)_{\varepsilon}=\left(\bar{p}_{\varepsilon}\right)_{x},\left(\bar{q}_{x}\right)_{\varepsilon}=\left(\bar{q}_{\varepsilon}\right)_{x}$, it follows that $\left\|\left(\bar{p}_{\varepsilon}^{2}+\bar{q}_{\varepsilon}^{2}\right)_{x}\right\|_{L^{1}} \rightarrow 0$ as $\varepsilon \rightarrow 0$. Therefore,

$$
\begin{aligned}
\left\|\left(\tilde{p}_{\varepsilon}\right)_{x}-\bar{p}_{x}\right\|_{L^{1}(\Omega)} \leq & \sup \left|\frac{\bar{p}_{\varepsilon}}{\left(\bar{p}_{\varepsilon}^{2}+\bar{q}_{\varepsilon}^{2}\right)^{\frac{3}{2}}}\right|\left\|\left(\bar{p}_{\varepsilon}^{2}+\bar{q}_{\varepsilon}^{2}\right)_{x}\right\|_{L^{1}(\Omega)} \\
& +\sup \left|\frac{1}{\sqrt{\bar{p}_{\varepsilon}^{2}+\bar{q}_{\varepsilon}^{2}}}\right|\left\|\left(\bar{p}_{x}\right)_{\varepsilon}-\bar{p}_{x}\right\|_{L^{1}(\Omega)} \rightarrow 0 .
\end{aligned}
$$

Similarly, $\left\|\left(\tilde{q}_{\varepsilon}\right)_{y}-\bar{q}_{y}\right\|_{L^{1}(\Omega)} \rightarrow 0$ and we conclude $\left\|D_{\tilde{v}} \tilde{p}_{\varepsilon}\right\|_{L^{1}(\Omega)} \rightarrow 0$ as $\varepsilon \rightarrow 0$. Hence, there exists a function $C(\varepsilon) \rightarrow 0$ as $\varepsilon \rightarrow 0$ so that

$$
\int_{\Omega}\left|D_{\tilde{v}} \tilde{p}_{\varepsilon}\right| \leq C(\varepsilon)
$$

So, applying the computation at the beginning of the proof with $f=\tilde{p}_{\varepsilon}$, we have

$$
\int_{V}\left|D_{\tilde{v}}^{h} \tilde{p}_{\varepsilon}\right| \leq C(\varepsilon)
$$

Thus, as $\varepsilon \rightarrow 0, D_{\tilde{v}}^{h} \tilde{p}_{\varepsilon} \rightarrow 0$ for almost every $x_{0} \in V$. To complete the proof, we would like to have that $\lim _{\varepsilon \rightarrow 0} D_{\tilde{v}}^{h} \tilde{p}_{\varepsilon}=D_{v}^{h} \bar{p}$. Assuming this for a moment, this would imply that $D_{v}^{h} \bar{p}=0$ almost everywhere as well. Then, taking a countable dense sequence $h_{n} \rightarrow 0$ and the countable intersection of full measure sets where $D_{v}^{h_{n}} \bar{p}=0$, we have a full measure subset of $V$, denoted by $V_{0}$, where

$$
D_{v}^{h_{n}} \bar{p}\left(x_{0}\right)=0 \quad \text { for all } n \in \mathbb{Z}_{+}, x_{0} \in V_{0} .
$$

By the continuity of $\bar{p}$ on this region, this implies that

$$
D_{v}^{h} \bar{p}(x)=0 \text { for all } h<\operatorname{dist}(x, \partial \Omega), x \in V .
$$


Thus we are left with verifying that

$$
\lim _{\varepsilon \rightarrow 0} D_{\tilde{v}}^{h} \tilde{p}_{\varepsilon}=D_{v}^{h} \bar{p}
$$

First we note that $D_{\tilde{v}}^{h} \tilde{p}_{\varepsilon}=D_{\tilde{v}}^{h} \bar{p}+o_{\varepsilon}(1)$ and that the convergence is uniform as $\tilde{p}_{\varepsilon} \rightarrow \bar{p}$ uniformly on $V$. So we merely need to verify that

$$
\lim _{\varepsilon \rightarrow 0} D_{\tilde{v}}^{h} \bar{p}=D_{v}^{h} \bar{p}
$$

To calculate the value of the limit, we will construct a sequences of integral curves using the work in appendix A. Letting $\Omega=V, X=(\bar{p}, \bar{q})$ and $X_{k}=\left(\tilde{p}_{\varepsilon_{k}}, \tilde{q}_{\varepsilon_{k}}\right)$ for a sequence $\varepsilon_{k} \rightarrow 0$, we apply the construction in appendix A to form appropriate integral curves for these vector fields. Then Lemma A.4 implies that $\bar{p}\left(c_{x_{0}}^{X_{k}}\right)(h) \rightarrow$ $\bar{p}\left(c_{x_{0}}^{X}(h)\right)$ and hence (8) is true.

Remark 2. We note that if we assume $v=\left(\frac{\bar{q}}{\bar{p}},-1\right)$ and that $S$ is a noncharacteristic patch of surface where $v$ is continuous, essentially the same argument proves that

$$
D_{v}^{h} \bar{q}(x)=0
$$

for $x \in V$ and $h<\operatorname{dist}(x, \partial \Omega)$. We note that since $\bar{p}^{2}+\bar{q}^{2}=1$, if $D_{v}^{h} \bar{q}=0$ implies that $D_{v}^{h} \bar{p}=0$ as well.

Lemma 3.3. If $S$, a $C^{1} H$-minimal surface, is decomposed as $N \cup \bigcup_{i=1}^{\infty} K_{i}$, then on each $K_{i}$ with nontrivial interior and with $\bar{p}, \bar{q} \in W^{1,1}$ the integral curves of $\boldsymbol{v}_{X}{ }^{\perp}$ are straight lines.

Proof. Let $\Omega_{1} \subset K_{i}$ be the open set where $\bar{q} \neq 0$ and let $\Omega_{2} \subset K_{i}$ be the set where $\bar{p} \neq 0$. Then $\Omega_{1} \cup \Omega_{2}=K_{i}$. Let $V_{j}$ be compactly contained in $\Omega_{j}$. By the previous lemma, since $D_{v}^{h} \bar{p}=0$ on $V_{1}$, we have that for each integral curve, $\mathcal{L}$ of $\boldsymbol{v}_{X}{ }^{\perp}, D_{v}^{h} \bar{p}$ is zero on almost every point of $\mathscr{L}$. Thus, for these integral curves $\boldsymbol{v}_{X}{ }^{\perp}=(\bar{q},-\bar{p})$ is constant almost everywhere along its own integral curves (which are the same as the integral curves of $v$ ). Thus, these integral curves are straight lines except potentially on a set of measure zero. However, the structure theorem of Franchi, Serapioni and Serra Cassano tells that $p$ is a continuous function and hence, $\bar{p}$ is discontinuous only at characteristic points. As the $K_{i}$ have no characteristic points, we see that $\bar{p}$ is continuous on each $K_{i}$. So, the integral curves are $C^{1}$ and thus, since they are lines almost everywhere, they must simply be straight lines. Similarly, the integral curves of $v_{X}^{\perp}$ are straight lines on $V_{2}$ as well using Remark 2. Using a compact exhaustion of the $\Omega_{i}$, we see that the integral curves of $v_{X}^{\perp}$ on $K_{i}$ are straight lines.

This is the same basic result we found in Section 4 of [12] - the integral curves of $\boldsymbol{v}_{X}{ }^{\perp}$ are straight lines. So, if we can construct a seed curve $\gamma$ as an integral curve of 
the vector field $\boldsymbol{v}_{X}$ and repeat the proof of Theorem 4.5 in [12] (this is Theorem 1.1 of the introduction), we have the same result for these $C^{1}$ noncharacteristic H-minimal graphs:

Proposition 3.4. Let $S \subset \mathbb{H}^{1}$ be a noncharacteristic patch of a $C^{1} H$-minimal surface of the type

$$
S=\left\{(x, y, t) \in \mathbb{H}^{1} \mid(x, y) \in \Omega, t=h(x, y)\right\},
$$

with $\bar{p}, \bar{q} \in W^{1,1}(\Omega)$ and where $h: \Omega \rightarrow \mathbb{R}$ is a $C^{k}$ function over a domain $\Omega$ in the $x y$-plane. Then, there exists a $C^{1}$ seed curve $\gamma$ so that $S$ can be locally parameterized by

$$
(s, r) \rightarrow\left(\gamma_{1}(s)+r \gamma_{2}^{\prime}(s), \gamma_{2}(s)-r \gamma_{1}^{\prime}(s), h(s, r)\right),
$$

where

$$
h(s, r)=h_{0}(s)-\frac{r}{2}\left\langle\gamma(s), \gamma^{\prime}(s)\right\rangle .
$$

and $h_{0}(s)=\left.h\right|_{\gamma(s)}$.

We note that if we knew $\gamma \in C^{2}$, the argument used to prove Theorem A in [12] would extend completely to this case, showing that a $C^{1}$ noncharacteristic graph is an $\mathrm{H}$-minimal surface if and only if it has such a representation for a neighborhood of each point on the surface. A priori, $\gamma$ is merely $C^{1}$ and need not have any higher regularity. However, we shall see that if $\Omega$ is "large in horizontal directions" then $\gamma^{\prime}$ is indeed $C^{1}$. To make this precise, we need a definition.

Definition 3.5. Suppose an open set $\Omega$ is parametrized by

$$
F(s, r)=\left(\gamma_{1}(s)+r \gamma_{2}^{\prime}(s), \gamma_{2}(s)-r \gamma_{1}^{\prime}(s)\right)
$$

where $\gamma$ is a seed curve. Let

$$
\begin{array}{r}
d(s)=\min \left\{\sup \left\{r_{1}\left|r_{1}>0, F(s, r)\right|_{r \in\left(0, r_{1}\right)} \in \Omega\right\},\right. \\
\left.\sup \left\{r_{2}\left|r_{2}>0, F(s, r)\right|_{r \in\left(-r_{2}, 0\right)} \in \Omega\right\}\right\}
\end{array}
$$

and let

$$
d(\Omega, \gamma)=\inf \{d(s) \mid \gamma(s) \in \Omega\}
$$

Lemma 3.6. Fix $\varepsilon>0$. Let $S$ be a $C^{1}$ noncharacteristic $H$-minimal graph defined over a planar domain $\Omega$ with $\bar{p}, \bar{q} \in W^{1,1}(\Omega)$ and let $\gamma$ be a seed curve for $S$. If $d(\Omega, \gamma)>\varepsilon$, then $\gamma^{\prime}(s)$ is locally Lipschitz.

Proof. We argue by contradiction. Suppose $\gamma^{\prime}(s)$ is not Lipschitz at $s=s_{0}$. Then, there exists a sequence $\left\{h_{n}\right\}$ tending to zero with

$$
\frac{\left|\gamma^{\prime}\left(s_{0}+h_{n}\right)-\gamma^{\prime}\left(s_{0}\right)\right|}{h_{n}}=\sqrt{2 L_{n}}
$$


with $L_{n} \rightarrow \infty$ as $n \rightarrow \infty$. Now

$\left|\gamma^{\prime}\left(s_{0}+h_{n}\right)-\gamma^{\prime}\left(s_{0}\right)\right|^{2}=\left(\gamma^{\prime}\left(s_{0}+h_{n}\right)-\gamma^{\prime}\left(s_{0}\right)\right) \cdot\left(\gamma^{\prime}\left(s_{0}+h_{n}\right)-\gamma^{\prime}\left(s_{0}\right)\right)=2-2 \cos \left(\theta_{n}\right)$.

Where $\theta_{n}$ is the angle between $\gamma^{\prime}\left(s_{0}\right)$ and $\gamma^{\prime}\left(s_{0}+h_{n}\right)$. So we must have that

$$
\frac{1-\cos \left(\theta_{n}\right)}{h_{n}^{2}}=L_{n}
$$

Rearranging, we have

$$
\cos \left(\theta_{n}\right)=1-h_{n}^{2} L_{n}
$$

Recalling that, by Proposition 3.4, $\boldsymbol{v}_{X}$ is constant along integral curves of $\boldsymbol{v}_{X}{ }^{\perp}$, for $\boldsymbol{v}_{X}$ to be well defined on $\Omega$, no two integral curves of $\boldsymbol{v}_{X}{ }^{\perp}$ may cross inside $\Omega$. Indeed, it two such curves crossed, then infinitely many of them would cross and we would have conflicting values for $\boldsymbol{v}_{X}$.

Next we use this to gain an estimate on $L_{n}$. Referring to Figure 1, we see that $\sin \left(\theta_{n}\right)=\frac{c}{a} \leq \frac{\left|\gamma\left(s_{0}+h_{n}\right)-\gamma\left(s_{0}\right)\right|}{a}$. Now, since $d(\Omega)>\varepsilon$, we have

$$
\begin{aligned}
\varepsilon & <d(\Omega) \leq a \\
& \leq \frac{\left|\gamma\left(s_{0}+h_{n}\right)-\gamma\left(s_{0}\right)\right|}{\sin \left(\theta_{n}\right)} \\
& =\frac{\left|\gamma\left(s_{0}+h_{n}\right)-\gamma\left(s_{0}\right)\right|}{\sqrt{1-\left(1-h_{n}^{2} L_{n}\right)^{2}}} \quad \text { (by equation (11)). }
\end{aligned}
$$

Thus we have

$$
\sqrt{1-\left(1-h_{n}^{2} L_{n}\right)^{2}} \leq \frac{\left|\gamma\left(s_{0}+h_{n}\right)-\gamma\left(s_{0}\right)\right|}{\varepsilon}
$$

Or, after some algebraic simplification,

$$
L_{n} \leq \frac{\left|\gamma\left(s_{0}+h_{n}\right)-\gamma\left(s_{0}\right)\right|^{2}}{\varepsilon^{2} h_{n}^{2}\left(1+\sqrt{1-\frac{\left|\gamma\left(s_{0}+h_{n}\right)-\gamma\left(s_{0}\right)\right|^{2}}{\varepsilon^{2}}}\right)} \rightarrow \frac{1}{2 \varepsilon^{2}} .
$$

But, by assumption, $L_{n} \rightarrow \infty$ as $n \rightarrow \infty$, so we reach a contradiction.

Theorem 3.7. Let $S$ be a $C^{1} H$-minimal graph over a domain $\Omega$ of the $x y$-plane with $\bar{p}, \bar{q} \in W^{1,1}(\Omega)$. If $\gamma$ is a seed curve for $S$ and $d(\Omega, \gamma)>\varepsilon>0$, then $\gamma$ is $C^{2}$.

Proof. By the previous lemma we know that $\gamma^{\prime}$ is Lipschitz and hence $\gamma^{\prime \prime}(s)$ exists for almost every $s$. Now consider a parameter value $s_{0}$ and sequences $\left\{s_{i}^{+}\right\}$and $\left\{s_{i}^{-}\right\}$so that $s_{i}^{+} \rightarrow s_{0}, s_{i}^{-} \rightarrow s_{0}$ and both $\gamma^{\prime \prime}\left(s_{i}^{+}\right)$and $\gamma^{\prime \prime}\left(s_{i}^{-}\right)$exist for all $i$. By the Lipschitz 
Vol. 81 (2006)

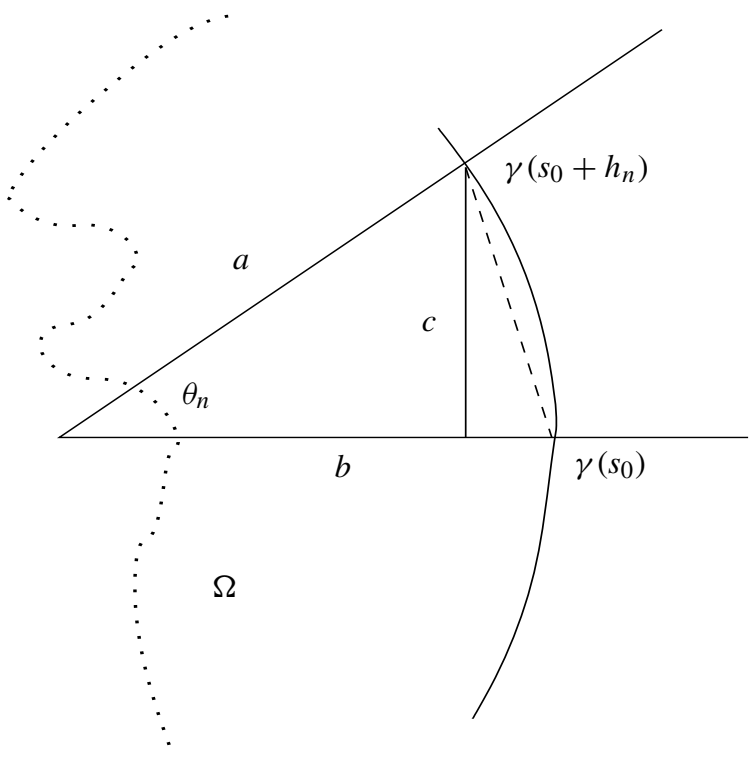

Figure 1. Illustration for Lemma 3.6.

condition on $\gamma^{\prime}$, we can always find such sequences and we may also assume, picking subsequences if necessary, that $\lim _{i \rightarrow \infty} \gamma^{\prime \prime}\left(s_{i}^{+}\right)$and $\lim _{i \rightarrow \infty} \gamma^{\prime \prime}\left(s_{i}^{-}\right)$both exist. Then we claim that

$$
\lim _{i \rightarrow \infty} \gamma^{\prime \prime}\left(s_{i}^{+}\right)=\lim _{i \rightarrow \infty} \gamma^{\prime \prime}\left(s_{i}^{-}\right) .
$$

To show this, we examine the Riemannian normal of the surface. As the surface is $C^{1}$, the normal must be continuous. We will show that if

$$
\lim _{i \rightarrow \infty} \gamma^{\prime \prime}\left(s_{i}^{+}\right) \neq \lim _{i \rightarrow \infty} \gamma^{\prime \prime}\left(s_{i}^{-}\right)
$$

then the normal cannot be continuous.

First, we a direct calculation using the representation of $S$ by

$$
\left(F(s, r), h_{0}(s)-\frac{r}{2} \gamma \cdot \gamma^{\prime}(s)\right)
$$

yields that the vector

$$
\eta(s, r)=\gamma_{1}^{\prime}(s) X_{1}+\gamma_{2}^{\prime}(s) X_{2}+\beta(s, r) T
$$

points in the direction of the Riemannian normal where

$$
\beta(s, r)=\frac{-1+r \kappa(s)}{h_{0}^{\prime}(s)-r+\frac{1}{2} \gamma^{\prime} \cdot \gamma^{\perp}+\frac{r^{2}}{2} \kappa(s)} .
$$


We note that this computation is contained in Section 4 of [12]. Now, by assumption, both $\gamma$ and $\gamma^{\prime}$ are continuous. To argue by contradiction, we assume that

$$
\lim _{i \rightarrow \infty} \gamma^{\prime \prime}\left(s_{i}^{+}\right)=l_{1}
$$

and

$$
\lim _{i \rightarrow \infty} \gamma^{\prime \prime}\left(s_{i}^{-}\right)=l_{2}
$$

where $l_{1} \neq l_{2}$. Let

$$
\kappa_{1}=\lim _{i \rightarrow \infty} \gamma^{\prime \prime}\left(s_{i}^{+}\right) \cdot \gamma^{\prime}\left(s_{i}^{+}\right)^{\perp}=l_{1} \cdot \gamma^{\prime}\left(s_{0}\right)^{\perp}
$$

and

$$
\kappa_{2}=\lim _{i \rightarrow \infty} \gamma^{\prime \prime}\left(s_{i}^{-}\right) \cdot \gamma^{\prime}\left(s_{i}^{-}\right)^{\perp}=l_{2} \cdot \gamma^{\prime}\left(s_{0}\right)^{\perp} .
$$

Now, looking at $\eta(s, r)$ along the two sequences, we know that the $X_{1}$ and $X_{2}$ components match as we tend towards $s_{0}$ as $\gamma^{\prime}$ is continuous. If $\eta$ is to be continuous, then $\beta$ must be continuous as well, i.e.

$$
\frac{-1+r \kappa_{1}}{h_{0}^{\prime}\left(s_{0}\right)-r+\frac{1}{2} \gamma^{\prime} \cdot \gamma^{\perp}+\frac{r^{2}}{2} \kappa_{1}}=\frac{-1+r \kappa_{2}}{h_{0}^{\prime}\left(s_{0}\right)-r+\frac{1}{2} \gamma^{\prime} \cdot \gamma^{\perp}+\frac{r^{2}}{2} \kappa_{2}} .
$$

After simplifying algebraically, this yields

$$
\left(\kappa_{1}-\kappa_{2}\right)\left(r h_{0}^{\prime}\left(s_{0}\right)+\frac{r}{2} \gamma^{\prime} \cdot \gamma^{\perp}-\frac{r^{2}}{2}\right)=0 .
$$

As $r$ can vary, we see that $\kappa_{1}=\kappa_{2}$. Since $\gamma$ is parameterized by arclength, we have that $\gamma^{\prime \prime} \cdot \gamma^{\prime}=0$ where defined. Combining this with equations (14) and (15) we reach a contradiction of the assumption that $l_{1} \neq l_{2}$. So we see that, where it is defined, $\gamma^{\prime \prime}$ coincides with a continuous function. Consider a point $s_{0}$ where, a priori, $\gamma^{\prime}$ is not differentiable. Then in a neighborhood $N$ of $s_{0}$ there is a full measure subset $N_{0}$ so that if $s \in N_{0}, \gamma^{\prime \prime}(s)$ exists. Then, as $\gamma^{\prime \prime}$ coincides with a continuous function where is exists, we see that

$$
\lim _{s \in N_{0}, s \rightarrow s_{0}} \frac{\gamma^{\prime}\left(s_{0}\right)-\gamma^{\prime}(s)}{s_{0}-s}
$$

exists. In other words, $s_{0}$ is a point of approximate differentiability for $\gamma^{\prime}$. Since, by the previous lemma, $\gamma^{\prime}$ is Lipschitz, Lemma 3.1.5 in [8] implies that $\gamma^{\prime}$ is differentiable at $s_{0}$ as well.

We combine the previous lemmas:

Theorem 3.8. Fix $\varepsilon>0$. Suppose $S$ is a $C^{1}$ noncharacteristic H-minimal graph over a region $\Omega$ with $\bar{p}, \bar{q} \in W^{1,1}(\Omega)$. If $\gamma$ is a seed curve of $S$ with $d(\Omega, \gamma)>\varepsilon$, then $\gamma \in C^{2}$. 
Remark 3. The previous theorem is a type of regularity result for H-minimal surfaces. Recalling that $\boldsymbol{v}_{X}=\left(\gamma_{1}^{\prime}(s), \gamma_{2}^{\prime}(s)\right)$, the theorem says that the vector field $\boldsymbol{v}_{X}$ is continuously differentiable. Therefore, the arguments from [12] used to prove Theorem 1.1 (this is Theorem A in [12]) apply and the surface can be realized by

$$
\left(\gamma_{1}(s)+r \gamma_{2}^{\prime}(s), \gamma_{2}(s)-r \gamma_{1}^{\prime}(s), h_{0}(s)-\frac{r}{2} \gamma \cdot \gamma^{\prime}(s)\right) .
$$

Moreover, the smoothness of such a piece of H-minimal surface is completely determined by the function $h_{0}(s)$. Given the structure theorem of Franchi et al., if the surface is an X-perimeter minimizer, the function $h_{0}(s)$ must be at least $C^{1}$ on the sets $K_{i}$.

We end this section by summarizing the results.

Theorem 3.9. If $S$ is an open $C^{1} H$-minimal graph over a domain $\Omega \subset \mathbb{R}^{2}$ with no characteristic points with unit horizontal Gauss map $\boldsymbol{v}_{X}$ in $W^{1,1}(\Omega)$, then the integral curves of $\boldsymbol{v}_{X}{ }^{\perp}$ are straight lines and $S$ can be locally parameterized by

$$
(s, r) \rightarrow\left(\gamma_{1}(s)+r \gamma_{2}^{\prime}(s), \gamma_{2}(s)-r \gamma_{1}^{\prime}(s), h(s, r)\right),
$$

where

$$
h(s, r)=h_{0}(s)-\frac{r}{2}\left\langle\gamma(s), \gamma^{\prime}(s)\right\rangle
$$

and $\gamma$ is an integral curve of $\boldsymbol{v}_{X}$. Moreover, if there exists $\varepsilon>0$ so that $d(\Omega, \gamma)>$ $\varepsilon>0$ then $h_{0}(s) \in C^{1}$ and $\gamma(s) \in C^{2}$.

This is Theorem A in the introduction.

We note that, as $\gamma \in C^{2}$, all of the computations of Section 4 of [12] are valid so long as they do not involve more than one derivative of $h_{0}(s)$. In particular, we have:

Proposition 3.10. Let $S$ be a patch of $C^{1} H$-minimal surface given by

$$
(s, r) \rightarrow\left(\gamma_{1}(s)+r \gamma_{2}^{\prime}(s), \gamma_{2}(s)-r \gamma_{1}^{\prime}(s), h(s, r)\right),
$$

where

$$
h(s, r)=h_{0}(s)-\frac{r}{2}\left\langle\gamma(s), \gamma^{\prime}(s)\right\rangle
$$

with $s \in\left(s_{0}, s_{1}\right), r \in\left(r_{0}, r_{1}\right)$. Then $S$ may be extended to a surface $\tilde{S}$ by including all portions of the rules, i.e. extending the above parameterization to $r \in(-\infty, \infty)$. In this case the surface $\tilde{S}$ has characteristic points at

$$
h_{0}^{\prime}(s)-r+\frac{1}{2} \gamma^{\prime}(s) \cdot \gamma(s)^{\perp}+\frac{r^{2}}{2} \kappa(s)=0 .
$$


Proof. The only new portion of this proposition is the identification of the characteristic locus. We note that by hypothesis, $d(\Omega, \gamma)=r_{1}-r_{0}>0$ and so $\gamma \in C^{2}$. As we assume the surface is $C^{1}$, we must have that $h_{0} \in C^{1}$ as well. To verify the position of the characteristic locus, we repeat the arguments found in [12], in particular the computations in the proof of Theorem A. We review them here for completeness. We first compute tangent vectors to the surface at each point by taking the $s$ and $r$ derivatives of the parameterization:

$$
\begin{gathered}
\tau=\frac{\partial}{\partial r}\left(\gamma_{1}(s)+r \gamma_{2}^{\prime}(s), \gamma_{2}(s)-r \gamma_{1}^{\prime}(s), h(s, r)\right) \\
=\left(\gamma_{2}^{\prime}(s),-\gamma_{1}^{\prime}(s),-\frac{1}{2}\left\langle\gamma(s), \gamma^{\prime}(s)\right\rangle\right) \\
=\gamma_{1}^{\prime}(s) X_{1}+\gamma_{2}^{\prime}(s) X_{2}, \\
\sigma=\frac{\partial}{\partial s}\left(\gamma_{1}(s)+r \gamma_{2}^{\prime}(s), \gamma_{2}(s)-r \gamma_{1}^{\prime}(s), h(s, r)\right) \\
=\left(\gamma_{1}^{\prime}(s)+r \gamma_{2}^{\prime \prime}(s), \gamma_{2}^{\prime}(s)-r \gamma_{1}^{\prime \prime}(s), h_{0}^{\prime}(s)-\frac{r}{2}-\frac{r}{2}\left\langle\gamma(s), \gamma^{\prime \prime}(s)\right\rangle\right) \\
=\left(\gamma_{1}^{\prime}(s)+r \gamma_{2}^{\prime \prime}(s)\right) X_{1}+\left(\gamma_{2}^{\prime}(s)-r \gamma_{1}^{\prime \prime}(s)\right) X_{2}+\left(h_{0}^{\prime}(s)-r\right. \\
\left.+\frac{1}{2}\left\langle\gamma^{\prime}(s), \gamma(s)^{\perp}\right\rangle+\frac{r^{2}}{2} \kappa(s)\right) T .
\end{gathered}
$$

Taking the cross product of these vectors with respect to the Riemannian structure, we have

$$
\sigma \times \tau=\gamma_{1}^{\prime}(s) B(s, r) X_{1}+\gamma_{2}^{\prime}(s) B(s, r) X_{2}+(-1+r \kappa(s)) T
$$

where

$$
B(s, r)=h_{0}^{\prime}(s)-r+\frac{1}{2}\left\langle\gamma^{\prime}(s), \gamma(s)^{\perp}\right\rangle+\frac{r^{2}}{2} \kappa(s) .
$$

As characteristic points arise when Riemannian normal, $\sigma \times \tau$, has only a $T$ component, we have the desired description of characteristic points.

We often use the notation

$$
W_{0}(s)=h_{0}^{\prime}(s)+\frac{1}{2}\left\langle\gamma^{\prime}(s), \gamma(s)^{\perp}\right\rangle .
$$

We end the section with an observation concerning the nature of the characteristic locus along a single rule. Equation (7) shows that, generically, each rule contains two characteristic points at

$$
r=\frac{1}{\kappa(s)} \pm \frac{\sqrt{1-2 \kappa(s) W_{0}(s)}}{\kappa(s)}
$$


one to each side of the point at $r=\frac{1}{\kappa(s)}$. In the special case where $W_{0}(s)=\frac{1}{2 \kappa(s)}$ we have a double characteristic point at $r=\frac{1}{\kappa(s)}$.

Lemma 3.11. Let $S$ be a $C^{1} H$-minimal graph parameterized by

$$
\left(F(s, r), h_{0}(s)-\frac{r}{2} \gamma(s) \cdot \gamma^{\prime}(s)\right), \quad(s, r) \in \Omega \subset \mathbb{R}^{2} .
$$

Suppose $\left(s_{0}, r_{0}\right)$ and $\left(s_{1}, r_{1}\right)$ are points so that $F\left(s_{0}, r_{0}\right)=F\left(s_{1}, r_{1}\right)$. Then

$$
\left(F\left(s_{0}, r_{0}\right), h_{0}\left(s_{0}\right)-\frac{r_{0}}{2} \gamma\left(s_{0}\right) \cdot \gamma^{\prime}\left(s_{0}\right)\right)
$$

is a characteristic point of $S$.

Proof. Since we assume that $S$ is a graph over the $x y$-plane, we must have that

$$
\left(F\left(s_{0}, r_{0}\right), h_{0}\left(s_{0}\right)-\frac{r_{0}}{2} \gamma\left(s_{0}\right) \cdot \gamma^{\prime}\left(s_{0}\right)\right)=\left(F\left(s_{1}, r_{1}\right), h_{0}\left(s_{1}\right)-\frac{r_{1}}{2} \gamma\left(s_{1}\right) \cdot \gamma^{\prime}\left(s_{1}\right)\right) .
$$

We recall that the unit horizontal Gauss map on $S$ is given by $\boldsymbol{v}_{X}(s, r)=\left(\gamma_{1}^{\prime}(s), \gamma_{2}^{\prime}(s)\right)$ and that the unit horizontal Gauss map is constant along any rule. The vector

$$
\eta(s, r)=\frac{\gamma_{1}^{\prime}(s)}{\sqrt{1+\beta(s, r)^{2}}} X_{1}+\frac{\gamma_{2}^{\prime}(s)}{\sqrt{1+\beta(s, r)^{2}}} X_{2}+\frac{\beta(s, r)}{\sqrt{1+\beta(s, r)^{2}}} T
$$

where

$$
\beta(s, r)=\frac{-1+r \kappa(s)}{W_{0}(s)-r+\frac{r^{2}}{2} \kappa(s)}
$$

points in the same direction as the unit Riemannian normal to the surface and is a completion of the unit horizontal Gauss map. As the surface is $C^{1}$, we must have that $\lim _{r \rightarrow r_{0}} \eta\left(s_{0}, r\right)=\lim _{r \rightarrow r_{1}} \eta\left(s_{1}, r\right)$. Since we assume the two rules are not parallel (they intersect), we have that $\gamma^{\prime}\left(s_{0}\right) \neq \gamma^{\prime}\left(s_{1}\right)$ and hence, for these limits to be equal, we must have that

$$
\lim _{r \rightarrow r_{i}} \beta\left(s_{i}, r\right)= \pm \infty .
$$

Examining the formula for the denominator of $\beta$ and equation (7), we see that the intersection must be a characteristic point.

Lemma 3.12. Let $S$ be a $C^{1} H$-minimal graph parameterized by

$$
\left(F(s, r), h_{0}(s)-\frac{r}{2} \gamma(s) \cdot \gamma^{\prime}(s)\right), \quad(s, r) \in \Omega \subset \mathbb{R}^{2} .
$$

Then along each rule, $\mathcal{L}_{\gamma(s)}(r)$, there is at most one characteristic point. 
Proof. As above, by equation (7), we see there are at most two characteristic points along $\mathcal{L}_{\gamma(s)}(r)$. Suppose there are two characteristic points along a rule $\mathscr{L}=$ $\mathcal{L}_{\gamma\left(s_{0}\right)}(r)$, one to each side of $r=\frac{1}{\kappa\left(s_{0}\right)}$. We claim that, arbitrarily close to $r=\frac{1}{\kappa\left(s_{0}\right)}$, $\mathcal{L}$ crosses another (nearby) rule. To see this, we first left translate and rotate the Heisenberg group so that $\gamma\left(s_{0}\right)=0$ and $\gamma^{\prime}\left(s_{0}\right)=(1,0)$ and reparametrize $\gamma$ so that $s_{0}=0$. From this normalization, we have that $F(0, r)=(0,-r)$. Consider a nearby rule, $\mathcal{L}_{\gamma\left(s_{1}\right)}(r)$. Then, direct calculation shows that

$$
F\left(s_{1},-\frac{\gamma_{1}\left(s_{1}\right)}{\gamma_{2}^{\prime}\left(s_{1}\right)}\right)=F\left(0, \gamma_{2}\left(s_{1}\right)+\frac{\gamma_{1}\left(s_{1}\right) \gamma_{1}^{\prime}\left(s_{1}\right)}{\gamma_{2}^{\prime}\left(s_{1}\right)}\right) .
$$

Taking that limit as $s_{1} \rightarrow 0$, we see that

$$
\gamma_{2}\left(s_{1}\right)+\frac{\gamma_{1}\left(s_{1}\right) \gamma_{1}^{\prime}\left(s_{1}\right)}{\gamma_{2}^{\prime}\left(s_{1}\right)} \rightarrow \frac{1}{\kappa(0)}
$$

Thus we make pick $s_{1}$ small enough so that

$$
\left.\mathcal{L}_{\gamma_{s_{1}}}(r) \cap \mathcal{L} \subset \mathcal{L}(r)\right|_{r \in\left(\frac{1}{\kappa(0)}-\varepsilon, \frac{1}{\kappa(0)}+\varepsilon\right)} .
$$

By the previous lemma, we see that there must be a characteristic point at this intersection distinct from the two characteristic points assumed to be along $\mathcal{L}$. This is a contradiction of (7), which shows that there are at most two characteristic points. Thus, along a rule contained in a piece of $\mathrm{H}$-minimal graph, there is at most a single characteristic point.

\section{Continuous H-minimal surfaces}

Again taking our motivation from the theorem of Franchi, Serapioni and Serra Cassano, we now investigate the possibility of gluing two pieces of different of $C^{1}$ $\mathrm{H}$-minimal surfaces together to form a new H-minimal surface from their union. In contrast to the classical cases, we can, under certain restrictions, create piecewise $C^{1}$ surfaces that are globally merely continuous and yet satisfy the H-minimal surface equation.

We consider the problem, discussed in the introduction, of gluing together two patches of $C^{1} \mathrm{H}$-minimal graphs so that the union satisfies the $\mathrm{H}$-minimal surface equation, at least weakly.

Proof of Theorem C. Assuming first that $S_{1} \cup S_{2}$ is H-minimal, we let

$$
\boldsymbol{v}_{X}=(\bar{p}, \bar{q})= \begin{cases}v_{1} & \text { on } \Omega_{1}, \\ v_{2} & \text { on } \Omega_{2}\end{cases}
$$


Then

$$
\int_{\Omega_{1} \cup \Omega_{2}} \bar{p} \varphi_{x}+\bar{q} \varphi_{y}=0
$$

for a smooth compactly supported (on $\Omega_{1} \cup \Omega_{2}$ ) test function $\varphi$. Recall that by Theorem 3.8, we know that $\left.\boldsymbol{v}_{X}\right|_{\tilde{\Omega}_{i}} \in C^{1}\left(\tilde{\Omega}_{i}\right)$. First we compute

$$
\begin{aligned}
\int_{\Omega_{i}} \bar{p}_{i} \varphi_{x}+\bar{q}_{i} \varphi_{y} & =\int_{\Omega_{i}}\left(\bar{p}_{i} \varphi\right)_{x}-\bar{p}_{i, x} \varphi+\left(\bar{q}_{i} \varphi\right)_{y}-\bar{q}_{i, y} \varphi \\
& =\int_{\Omega_{i}}\left(\bar{p}_{i} \varphi\right)_{x}+\left(\bar{q}_{i} \varphi\right)_{y}-\int_{\Omega_{i}} \bar{p}_{i, x} \varphi+\bar{q}_{i, y} \varphi \\
& =\int_{\Omega_{i}}\left(\bar{p}_{i} \varphi\right)_{x}+\left(\bar{q}_{i} \varphi\right)_{y} \\
& =\int_{\partial \Omega_{i}}-\bar{q}_{i} \varphi d x+\bar{p}_{i} \varphi d y \quad \text { (by Green's theorem) } \\
& =\int_{C}-\bar{q}_{i} \varphi d x+\bar{p}_{i} \varphi d y \\
& =\int_{C} \varphi\left(v_{i} \cdot \vec{n}_{i}\right) d s .
\end{aligned}
$$

The third equality holds because the surface over the interior of $\Omega_{i}$ satisfies the minimal surface equation. The second to last equality holds because $\varphi$ is compactly supported on $\Omega_{1} \cup \Omega_{2}$ and hence can only be nonzero on $C=\Omega_{1} \cap \Omega_{2}$. In the last equality, $\vec{n}_{i}$ denotes the inward pointing unit normal vector to $\partial \Omega_{i}$.

Applying this we have

$$
\begin{aligned}
\int_{\Omega_{1} \cup \Omega_{2}} \bar{p} \varphi_{x}+\bar{q} \varphi_{y} & =\int_{\Omega_{1}} \bar{p}_{1} \varphi_{x}+\bar{q}_{1} \varphi_{y}+\int_{\Omega_{2}} \bar{p}_{2} \varphi_{x}+\bar{q}_{2} \varphi_{y} \\
& =\int_{\partial \Omega_{1}} \varphi\left(v_{1} \cdot \vec{n}_{1}\right)+\int_{\partial \Omega_{2}} \varphi\left(v_{2} \cdot \vec{n}_{2}\right) \\
& =\int_{C} \varphi\left(v_{1} \cdot \vec{n}_{1}\right)+\int_{C} \varphi\left(v_{2} \cdot\left(-\vec{n}_{1}\right)\right) \\
& =\int_{C} \varphi\left(\left(v_{1}-v_{2}\right) \cdot \vec{n}_{1}\right) \\
& =0 .
\end{aligned}
$$

The second equality follows by the previous computation, where $\vec{n}_{i}$ are the inward pointing unit normal vectors. The differentiability of the $v_{i}$ and the fact that the $S_{i}$ are H-minimal implies that on the interior of $\Omega_{i}$, we have that $\bar{p}_{i, x}+\bar{q}_{i, y}=0$. In the third equality, we observe that $\varphi$ is zero on the boundary of $\Omega_{1} \cup \Omega_{2}$ and that on $C$, $\vec{n}_{1}=-\vec{n}_{2}$. 
Thus, we have that $\left(v_{1}-v_{2}\right) \cdot \vec{n}_{1}$ is weakly zero and hence, $v_{1}-v_{2}$ must be tangent to $C$ almost everywhere. Reversing the computation shows the sufficiency of this condition as well.

We illustrate this with an example where we glue two different $\mathrm{H}$-minimal surfaces along a rule.

Example 1. This theorem allows us to create many continuous H-minimal surfaces which are piecewise $C^{1}$. We illustrate how to use this theorem by constructing a new $\mathrm{H}$-minimal surface by gluing together the lower half of the plane $t=0$ with a portion of the surface $t=\frac{x y}{2}$ (see Figure 2). To do this we define the following seed curve:

$$
\gamma(s)= \begin{cases}(1, s) & 0 \leq s<\infty, \\ (\cos (s), \sin (s)) & -\pi \leq s<0, \\ (-1,-s-\pi) & -\infty<s<-\pi .\end{cases}
$$

Note that, as a plane curve, $\gamma$ is two vertical lines glued to the bottom half of a circle.

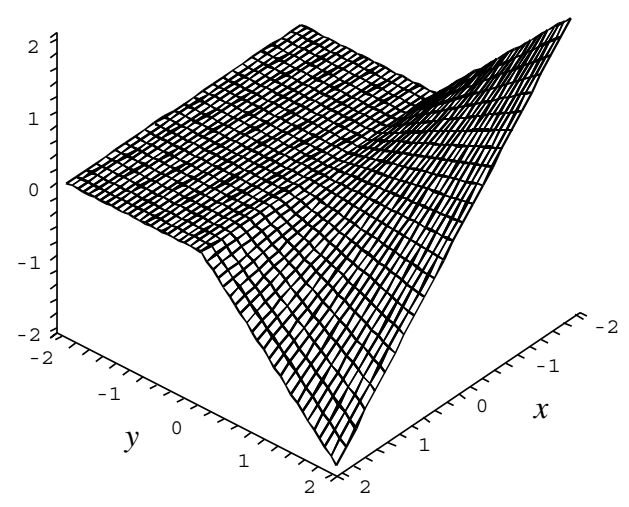

Figure 2

Now, we construct an H-minimal surface from this seed curve. With appropriate choices of $h_{0}(s)$, this yields the parameterization:

$$
S:= \begin{cases}\left(1+r, s,-\frac{s r}{2}-\frac{s}{2}\right) & 0 \leq s<\infty,-1 \leq r<\infty, \\ ((1+r) \cos (s),(1+r) \sin (s), 0) & -\pi<s<0,-1 \leq r<\infty, \\ \left(-1-r,-s-\pi,-\frac{r(s+\pi)}{2}-\frac{s+\pi}{2}\right) & -\infty<s \leq-\pi,-1 \leq r<\infty .\end{cases}
$$


Calculating in $x y$-coordinates, we find the unit horizontal Gauss map to be:

$$
\boldsymbol{v}_{X}=(\bar{p}, \bar{q})= \begin{cases}(0, \operatorname{sgn}(x)) & y \geq 0, \\ \left(-\frac{y}{\sqrt{x^{2}+y^{2}}}, \frac{x}{\sqrt{x^{2}+y^{2}}}\right) & y<0 .\end{cases}
$$

Direct computation shows that, away from $y=0$, these are H-minimal surfaces.

We note that, using the notation of the theorem, $v_{1}=v_{2}$ along the line $y=0$ and hence the hypotheses of the theorem are satisfied so long as we pick $\Omega_{1}$ and $\Omega_{2}$ away from the characteristic locus (for example, we might consider $\Omega_{1}=\{(x, y) \mid 2 \geq$ $x \geq 1,2 \geq y \geq 0\}$ and $\left.\Omega_{2}=\{(x, y) \mid 2 \geq x \geq 1,-2 \leq y \leq 0\}\right)$.

\section{5. $C^{\infty}$ solutions to the Plateau Problem: persistent H-minimal surfaces}

In [16], we showed that H-minimal graphs can arise as limits of minimal surfaces in $\left(\mathbb{H}^{1}, g_{\lambda}\right)$. In this section, we examine those surfaces which are minimal for all values of $\lambda \in[1, \infty)$.

Definition 5.1. A nonparametric graph is called a persistent H-minimal surface if it is $\mathrm{H}$-minimal and is minimal in $\left(\mathbb{H}^{1}, g_{\lambda}\right)$ for all $\lambda \in[1, \infty]$.

As shown in [16] Theorem 3.6 and 3.7 this implies that the surface is H-minimal and, for any curve satisfying the bounded slope condition that such a surface spans, it is the solution to the Plateau problem for this curve. Thus, the persistent minimal surfaces are a (small) class of smooth solutions to the Plateau problem. Moreover, as a consequence of Remark 1 in Section 3 of [16], we have the following characterization of persistent minimal surfaces.

Theorem 5.2. An H-minimal surface $S=\{(x, y, h(x, y))\}$ is persistent if and only if $\Delta h=0$ where $\Delta$ is the usual Laplacian in $\mathbb{R}^{2}$.

In this section, we will use the representation formula from Theorem 1.1 and some results from [12] to classify the persistent $\mathrm{H}$-minimal surfaces.

Lemma 5.3. If an H-minimal graph $S$ is persistent then the signed curvature of its seed curve is constant.

Proof. First we assume that $S$ is a persistent H-minimal graph. If $S$ is given by $(x, y, h(x, y))$ then $p=h_{x}-\frac{y}{2}$ and $q=h_{y}+\frac{x}{2}$ and so $\Delta h=0$ is equivalent to $p_{x}+q_{y}=0$. Using the notation from the previous section, we have $p=\alpha \bar{p}$ and 
$q=\alpha \bar{q}$ and so

$$
\begin{aligned}
p_{x}+q_{y} & =(\alpha \bar{p})_{x}+(\alpha \bar{q})_{y} \\
& =\nabla \alpha \cdot \boldsymbol{v}_{X}+\alpha\left(\bar{p}_{x}+\bar{q}_{y}\right) \\
& =\nabla \alpha \cdot \boldsymbol{v}_{X} \quad(\text { since } S \text { is H-minimal) } \\
& =0 \quad \text { (since we assume } S \text { is persistent). }
\end{aligned}
$$

Thus $\alpha$ is constant along the curves $F\left(s, t_{0}\right)$ and so we may write $\alpha(s, r)=\alpha(r)$. However, from Theorem 7.1 in [12], we know that

$$
\alpha(s, r)=\frac{\frac{\kappa(s)}{2} r^{2}-r+\alpha_{0}(s)}{1-\kappa(s) r} .
$$

Since $\alpha$ is constant along $F(s, 0)$ this implies that $\alpha_{0}(s) \equiv \alpha_{0}$ is constant, and so $\kappa(s)$ must also be constant.

Theorem 5.4. The persistent H-minimal graphs fall into two categories up to isometric transformations of $\left(\mathbb{H}^{1}, d_{\mathrm{CC}}\right)$ :

(1) $(\kappa=0)$

$$
\begin{aligned}
h(x, y)= & \frac{m}{1+m^{2}}\left(x-x_{0}\right)^{2}+\frac{m^{2}-1}{m^{2}+1}\left(x-x_{0}\right)\left(y-y_{0}\right)-\frac{m}{1+m^{2}}\left(y-y_{0}\right)^{2} \\
& +\frac{a}{\sqrt{1+m^{2}}}\left(x-x_{0}\right)+\frac{a m}{\sqrt{1+m^{2}}}\left(y-y_{0}\right)+b
\end{aligned}
$$

for $m, a, b \in \mathbb{R}$.

(2) $(\kappa \neq 0) S$, given in cylindrical coordinates, is

$$
(\rho \cos (\theta), \rho \sin (\theta), a \theta+b)
$$

for $a, b \in \mathbb{R}$.

Proof. By the previous lemma, we know that $\kappa$ must be constant for $S$ to be persistent.

Case 1. $\kappa=0$. If the curvature of the seed curve is zero, it is a line in the plane. By left translation, we may move the surface $S$ so that the seed curve passes through the origin. Thus, we may assume that

$$
\gamma(s)=\left(\frac{s}{\sqrt{1+m^{2}}}, \frac{m s}{\sqrt{1+m^{2}}}\right)
$$

for some $m \in \mathbb{R}$. Note that $\gamma(s) \cdot \gamma^{\prime}(s)=s$ In this case the parameterization $F$ is simply a linear transformation of the plane and we can write $(s, r)$ in terms of $(x, y)$. 
Indeed, we have

$$
\begin{aligned}
& s=\frac{x}{\sqrt{1+m^{2}}}+\frac{m y}{\sqrt{1+m^{2}}}, \\
& r=\frac{m x}{\sqrt{1+m^{2}}}-\frac{y}{\sqrt{1+m^{2}}} .
\end{aligned}
$$

Plugging this into the representation given in Theorem A, we get

$$
\begin{aligned}
h(x, y)= & h_{0}(s)+\frac{1}{2} r s \\
= & h_{0}\left(\frac{x}{\sqrt{1+m^{2}}}+\frac{m y}{\sqrt{1+m^{2}}}\right) \\
& \quad+\frac{1}{2}\left(\frac{m x}{\sqrt{1+m^{2}}}-\frac{y}{\sqrt{1+m^{2}}}\right)\left(\frac{x}{\sqrt{1+m^{2}}}+\frac{m y}{\sqrt{1+m^{2}}}\right) \\
= & h_{0}\left(\frac{x}{\sqrt{1+m^{2}}}+\frac{m y}{\sqrt{1+m^{2}}}\right)+\frac{m}{1+m^{2}} x^{2}+\frac{m^{2}-1}{m^{2}+1} x y-\frac{m}{1+m^{2}} y^{2} .
\end{aligned}
$$

Now, $S$ is persistent if and only if $\Delta h=0$,

$$
\begin{aligned}
h_{x x}+h_{y y} & =\left(h_{0}^{\prime \prime} \frac{1}{1+m^{2}}+2 \frac{m}{1+m^{2}}\right)+\left(h_{0}^{\prime \prime} \frac{m^{2}}{1+m^{2}}-2 \frac{m}{1+m^{2}}\right) \\
& =h_{0}^{\prime \prime} \\
& =0 .
\end{aligned}
$$

Thus, surfaces in this case are persistent if and only if $h_{0}$ is linear, i.e. $h_{0}=c s+d$ for some $a, b \in \mathbb{R}$. If we now left translate the resulting surfaces by a fixed basepoint $\left(x_{0}, y_{0}, t_{0}\right)$, we have that the surface is given by

$$
\begin{aligned}
(x, y, h(x, y))= & \left(x_{0}, y_{0}, t_{0}\right) \cdot\left(x, y, c\left(\frac{x}{\sqrt{1+m^{2}}}+\frac{m y}{\sqrt{1+m^{2}}}\right)\right. \\
& \left.+\frac{m}{1+m^{2}} x^{2}+\frac{m^{2}-1}{m^{2}+1} x y-\frac{m}{1+m^{2}} y^{2}+d\right) \\
= & \left(x+x_{0}, y+y_{0}, t_{0}+c\left(\frac{x}{\sqrt{1+m^{2}}}+\frac{m y}{\sqrt{1+m^{2}}}\right)\right. \\
& \left.+\frac{m}{1+m^{2}} x^{2}+\frac{m^{2}-1}{m^{2}+1} x y-\frac{m}{1+m^{2}} y^{2}+d+\frac{1}{2} x_{0} y-\frac{1}{2} y_{0} x\right) .
\end{aligned}
$$

Substituting $\bar{x}=x+x_{0}$ and $\bar{y}=y-y_{0}$ and collecting terms yields the claim. 
Case 2. $\kappa \neq 0$. If $\kappa=c \neq 0$, then $\gamma(s)$ must be a circle and after a suitable left translation, we may write

$$
\gamma(s)=\left(\frac{1}{c} \cos (s), \frac{1}{c} \sin (s)\right) .
$$

Hence $\gamma(s) \cdot \gamma^{\prime}(s)=0$. Moreover, the parameterization $F$ yields

$$
x=\left(r-\frac{1}{c}\right) \cos (s), \quad y=\left(r-\frac{1}{c}\right) \sin (s),
$$

and

$$
s=\arctan \left(\frac{y}{x}\right), \quad r=\sqrt{x^{2}+y^{2}}+\frac{1}{c} .
$$

Thus

$$
h=h_{0}(s)+\frac{r}{2} \gamma(s) \cdot \gamma^{\prime}(s)=h_{0}\left(\arctan \left(\frac{y}{x}\right)\right) .
$$

Computing the Laplacian of $h$ yields

$$
\Delta h=\frac{h_{0}^{\prime \prime}\left(\arctan \left(\frac{y}{x}\right)\right)}{x^{2}+y^{2}} .
$$

Thus, $\Delta h=0$ if and only if $h_{0}^{\prime \prime}(s)=0$ or that $h_{0}$ is linear. Thus, using cylindrical coordinates, $S$ is given by

$$
(\rho \cos (\theta), \rho \sin (\theta), a \theta+b)
$$

for $a, b \in \mathbb{R}$.

We record the observation made above:

Corollary 5.5. Any closed curve lying on the surfaces identified in Theorem 5.4 has a $C^{\infty}$ solution to the Plateau problem.

\section{Obstructions to H-minimal spanning surfaces of high regularity}

Throughout the rest of this paper, we will be considering a smooth closed curve

$$
c(\theta)=\left(c_{1}(\theta), c_{2}(\theta), c_{3}(\theta)\right) \subset \mathbb{H}^{1}
$$

with the property that $c(\theta)$ is a graph over the projection of $c$ to the $x y$-plane. For ease of notation, we will denote this projection by $\bar{c}(\theta)=\left(c_{1}(\theta), c_{2}(\theta), 0\right)$. When the context is clear, we suppress the last coordinate of the projection. We will be considering H-minimal spanning surfaces for these curves and moreover will consider only $C^{1} \mathrm{H}$-minimal spanning surfaces that are ruled graphs. To be precise, we make a definition: 
Definition 6.1. A $C^{1}$ ruled H-minimal graph, $S$, over a closed domain $\Omega \in \mathbb{R}^{2}$ is a ruled $\mathrm{H}$-minimal graph with the property that every rule in $S$ that meets a the characteristic locus may be extended over the characteristic locus as a straight line.

In other words, we will not consider gluings of the type discussed in Theorem C. We note that the work in [12] or [5] shows that $C^{2} \mathrm{H}$-minimal surfaces satisfy this definition.

If $c$ lies on a $C^{1}$ ruled $\mathrm{H}$-minimal graph then a geodesic line intersects each point on $c$ and, potentially, one or more other points on $c$ (see Figure 3). One easy way to determine the possible geodesic lines which are allowable for a specific point, $c\left(\theta_{0}\right)$, on the curve is to left translate that point to the origin (recalling that left translation preserves minimality). At the origin, the horizontal plane is the $x y$-plane and so,

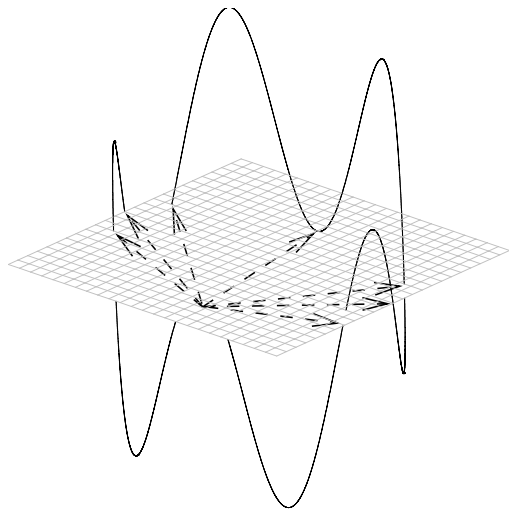

Figure 3. The dotted arrows are the possible rules emanating from the point.

points which can be connected to $c\left(\theta_{0}\right)$ by geodesic lines are those points on the left translated curve which lie on the $x y$-plane. Using the Campbell-Baker-Hausdorff formula, one can calculate this set explicitly as:

$$
A\left(\theta_{0}\right)=\left\{\theta \mid c_{3}(\theta)-c_{3}\left(\theta_{0}\right)-\frac{1}{2} c_{1}(\theta) c_{2}\left(\theta_{0}\right)+\frac{1}{2} c_{1}\left(\theta_{0}\right) c_{2}(\theta)=0\right\} .
$$

Note that $\theta_{0} \in A\left(\theta_{0}\right)$. In terms of building up a ruled surface which spans $c$, the larger $A\left(\theta_{0}\right)$, the more flexibility one has in developing a surface. On the other hand, if $A\left(\theta_{0}\right)$ contains only $\theta_{0}$ itself, this places great restriction on the possibilities of smooth spanning $\mathrm{H}$-minimal surfaces.

Consider a $C^{k}$ closed curve $c:\left[t_{0}, t_{1}\right) \rightarrow \mathbb{H}^{1}$ which is a graph over a curve, $\bar{c}$, in the $x y$-plane. Suppose $c$ is spanned by a ruled $\mathrm{H}$-minimal surface, $S$, which is a graph over a closed domain $\Omega$ in the $x y$-plane so that $\partial \Omega=\bar{c}$. Then the definition of 
$A$ above implicitly defines a function $\varphi(t)$ for $t \in\left[t_{0}, t_{1}\right)$ via the equation

$$
c_{3}(\varphi(t))-c_{3}(t)-\frac{1}{2} c_{1}(\varphi(t)) c_{2}(t)+\frac{1}{2} c_{1}(t) c_{2}(\varphi(t))=0 .
$$

As $c_{i} \in C^{k}, \varphi$ is also $C^{k}$. Moreover, we claim that such a $\varphi$ must be monotone. To see this, suppose that $\varphi$ is not monotone and that there exist $t_{0}, t_{1}, t_{2}$ so that $\varphi\left(t_{0}\right)=\varphi\left(t_{2}\right)$ and $\varphi\left(t_{1}\right) \neq \varphi\left(t_{0}\right)$. Let $\mathcal{L}_{i}$ be the rule connecting $c\left(t_{i}\right)$ to $c\left(\varphi\left(t_{i}\right)\right)$ and let $\overline{\mathcal{L}}_{i}$ be the projection of $\mathcal{L}_{i}$ to the $x y$-plane. Then, the assumption on $\varphi$ implies that $\overline{\mathscr{L}}_{1}$ intersects either $\overline{\mathcal{L}}_{2}$ or $\overline{\mathscr{L}}_{0}$. Without loss of generality, we will assume it intersects $\overline{\mathscr{L}}_{2}$. Further, $\overline{\mathscr{L}}_{2}$ must intersect the projection of every rule connecting $c(t)$ to $c(\varphi(t))$ for $t \in\left(t_{0}, t_{1}\right)$. Such intersection points must be characteristic points of the surface and so $\mathscr{L}_{2}$ would contain infinitely many characteristic points in violation of Lemma 3.12. We note that, given a monotone $C^{k}$ function $\varphi: S^{1} \rightarrow S^{1}$, it is easy to construct a $C^{k}$ ruled $\mathrm{H}$-minimal graph.

We record this observation:

Existence Criterion. Given a closed curve $c \in C^{k}$ which is a graph over a curve in the $x y$-plane, $c$ is spanned by a ruled $H$-minimal graph if and only if there exists $a$ monotone $C^{k}$ function $\varphi: S^{1} \rightarrow S^{1}$ with $\varphi(\theta) \in A(\theta)$.

Definition 6.2. A point $c\left(\theta_{0}\right)$ is called Legendrian if $c^{\prime}\left(\theta_{0}\right) \in \operatorname{span}\left\{X_{1}, X_{2}\right\}$. We call a point isolated if

$$
\left\{\theta_{0}\right\}=A\left(\theta_{0}\right)
$$

We record an immediate consequence of the definition.

Lemma 6.3. If $c(\theta)$ is an isolated point and $c$ is spanned by a ruled $H$-minimal graph, then $c(\theta)$ is Legendrian and the rule passing through $c(\theta)$ must be tangent to $c$.

Proof. If $c(\theta)$ is isolated then, by definition, it cannot be connected to another point of $c$ via a rule. As a consequence, we note that the projection a rule through $c(\theta)$ to the $x y$-plane cannot be transverse to $\bar{c}$. Indeed, if the projection were transverse, then it would intersect another point on $\bar{c}$. As $S$ is assumed to be a graph, this rule would then be forced to intersect another point on $c$. Now, consider the rule through $c(\theta)$. It is the limit of rules connecting points near $c(\theta)$. In other words, it is the limit of secant lines and hence must be a tangent line to $c$ and $c(\theta)$.

We next consider the relationship between two points on $c$ which are connected by a rule on a spanning $\mathrm{H}$-minimal surface.

Lemma 6.4. Suppose $c\left(\theta_{1}\right)$ and $c\left(\theta_{2}\right)$ are connected by a rule, $\mathcal{L}$, of a ruled surface spanning $c$. Then $c_{3}^{\prime}\left(\theta_{2}\right)$ is proportional to the third coordinate of the parallel translation of $c^{\prime}\left(\theta_{1}\right)$ along $\mathcal{L}$. The proportionality constant is given as the derivative at the point $c\left(\theta_{2}\right)$ of the parametrization of the curve induced by the ruling around $c\left(\theta_{2}\right)$. 
Proof. Without loss of generality, we may assume that $\theta_{1}=0$ and $c(0)=0$ via a reparametrization of $c$ and a composition with left translation in the Heisenberg group. By hypothesis, $A(0)$ contains the point $c\left(\theta_{2}\right)$ which, by abuse of notation, we will still identify by the parameter value $\theta_{2}$ despite having reparametrized the curve. Note, that under this renormalization, the rule $\mathcal{L}$ can be parametrized as

$$
\mathscr{L}(\tau)=\left(\tau c_{1}\left(\theta_{2}\right), \tau c_{2}\left(\theta_{2}\right), 0\right) .
$$

Moreover, the assumption that $c$ lies on a ruled surface implies that there exists a mapping $\varphi:(-\varepsilon, \varepsilon) \rightarrow\left(\theta_{2}-\delta, \theta_{2}+\delta\right)$ (with appropriately small $\varepsilon$ and $\delta$ ) so that $c(\varphi(t)) \in A(c(t))$ for $t \in(-\varepsilon, \varepsilon)$ and $c(t)$ is connected to $c(\varphi(t))$ by a rule. Thus, by the definition of $A(\theta)$ we have

$$
c_{3}(\varphi(t))-c_{3}(t)-\frac{1}{2} c_{1}(\varphi(t)) c_{2}(t)+\frac{1}{2} c_{1}(t) c_{2}(\varphi(t))=0 .
$$

Taking a derivative at $t=0$ and recalling that $c_{1}(0)=0=c_{2}(0)$ we get

$$
c_{3}^{\prime}\left(\theta_{2}\right) \varphi^{\prime}(0)=c_{3}^{\prime}(0)-\frac{1}{2} \bar{c}\left(\theta_{2}\right) \cdot\left(c_{2}^{\prime}(0),-c_{1}^{\prime}(0)\right) .
$$

Next we note that the vector field

$$
W=c_{1}^{\prime}(0) X_{1}+c_{2}^{\prime}(0) X_{2}+\left(c_{3}^{\prime}(0)+\frac{\tau}{2}\left(\bar{c}\left(\theta_{2}\right) \cdot\left(c_{2}^{\prime}(0),-c_{1}^{\prime}(0)\right)\right) T\right.
$$

is parallel along $\mathcal{L}$. Indeed, the tangent field to $\mathcal{L}$ is given by

$$
V=c_{1}\left(\theta_{2}\right) X_{1}+c_{2}\left(\theta_{2}\right) X_{2}
$$

and a direct computation shows that $V W=0$. Since the third coordinate of $W$ at $\tau=1$ is proportional to the expression in equation (28), we have the desired result.

Remark 4. Geometrically, this says that the height function, relative to translation in the Heisenberg group, remains constant along the rules. Thus, H-minimal surfaces are significantly more limited than ruled surfaces in Euclidean $\mathbb{R}^{3}$. A comparable class of ruled surfaces in $\mathbb{R}^{3}$ would be those ruled surfaces that contain only rules parallel to the $x y$-plane.

6.1. Curves with isolated points. We next turn to the problem of identifying curves that have ruled $\mathrm{H}$-minimal spanning surfaces. We begin with an investigation of curves that have isolated points.

Lemma 6.5. Suppose $c$ is a $C^{2}$ curve in $\mathbb{H}^{1}$ and $c\left(\theta_{0}\right)$ is an isolated point. Then there is an open neighborhood, $I=\left(\theta_{0}-\varepsilon, \theta_{0}+\delta\right)$, where $\left.c\right|_{I}$ sits on a piece of a ruled surface. 
Proof. Note that, without loss of generality, by composing with a left translation and reparametrizing the curve, we may assume that $\theta_{0}=0$ and that $c(0)=0$. We are attempting to parametrize a piece of the curve for $t \in(-\varepsilon, 0]$ in terms of parameter values $t \in[0, \delta)$ by associating a $\varphi(t) \in(-\varepsilon, 0]$ to $t \in[0, \delta)$. So, we will construct a $\operatorname{map} \varphi:[0, \delta) \rightarrow(-\varepsilon, 0]$ with $\varphi(0)=0$ so that $A(t)$ contains $\varphi(t)$. By the definition of $A$, this implies that

$$
c_{3}(\varphi(t))-c_{3}(t)-\frac{1}{2} c(\varphi(t)) \cdot c(t)^{\perp}=0 .
$$

Differentiating with respect to $t$ solving for $\varphi^{\prime}(t)$, we get

$$
\varphi^{\prime}(t)=\frac{c_{3}^{\prime}(t)+\frac{1}{2} c(\varphi(t)) \cdot c^{\prime}(t)^{\perp}}{c_{3}^{\prime}(\varphi(t))-\frac{1}{2} c^{\prime}(\varphi(t)) \cdot c(t)^{\perp}}=\frac{c_{3}^{\prime}(t)+\frac{1}{2} c(\varphi(t)) \cdot c^{\prime}(t)^{\perp}}{c_{3}^{\prime}(\varphi(t))+\frac{1}{2} c(t) \cdot c^{\prime}(\varphi(t))^{\perp}} .
$$

Note that, at $t=0$, recalling that $c(0)=0$, we see that

$$
\varphi^{\prime}(0)=\frac{c_{3}^{\prime \prime}(0)}{\varphi^{\prime}(0) c_{3}^{\prime \prime}(0)} \Longrightarrow \varphi^{\prime}(0)^{2}=1
$$

Thus, for at least a small time, $\varphi(t)$, defined implicitly by (29), exists and hence, for some interval $I$, there exists a ruled surface spanning $\left.c\right|_{I}$.

Remark 5. In the proof above, we see the obstruction - to be able to span a given curve with a ruled surface we must be able to find a function $\varphi$ describing how to connect points on $c$ via rules that is monotone.

Example 2. Consider the curve (see Figure 4a)

$$
c_{1}(\theta)=(1-\cos (\theta), \sin (\theta), 2-2 \cos (\theta)+\sin (\theta)-\sin (\theta) \cos (\theta))
$$

for $\theta \in[0,2 \pi)$. We quickly compute that

$$
\begin{aligned}
A\left(\theta_{0}\right)=\{\theta \mid & 2 \cos \left(\theta_{0}\right)+\frac{1}{2} \sin \left(\theta_{0}\right)+\sin \left(\theta_{0}\right) \cos \left(\theta_{0}\right)-2 \cos (\theta) \\
+ & \left.\frac{1}{2} \sin (\theta)-\sin (\theta) \cos (\theta)+\frac{1}{2}\left(\sin (\theta) \cos \left(\theta_{0}\right)-\sin \left(\theta_{0}\right) \cos (\theta)\right)\right\}
\end{aligned}
$$

and note that for $\theta_{0}=0$,

$$
A(0)=\{\theta \mid 2-2 \cos (\theta)+\sin (\theta)-\sin (\theta) \cos (\theta)=0\}=\{2 n \pi\} .
$$

Thus $\theta_{0}=0$ is an isolated point. Considering $\theta$ as a function of $\theta_{0}$, we see in Figure $4 \mathrm{~b}$ that there is another isolated point for $\theta_{0}$ slightly less than $\pi$. We will denote this value 
of $\theta_{0}$ by $\alpha$. Observing Figure $4 \mathbf{b}$, we see that for each $\theta_{0}=(0, \alpha)$ we can connect $c_{1}\left(\theta_{0}\right)$ to the unique point $c_{1}\left(\varphi\left(\theta_{0}\right)\right)$. Figure $4 \mathrm{c}$ illustrates several of the constructed rules connecting points on the curve and Figure $4 \mathrm{~d}$ shows the projections of Figure $4 \mathrm{c}$ to the $x y$-plane.

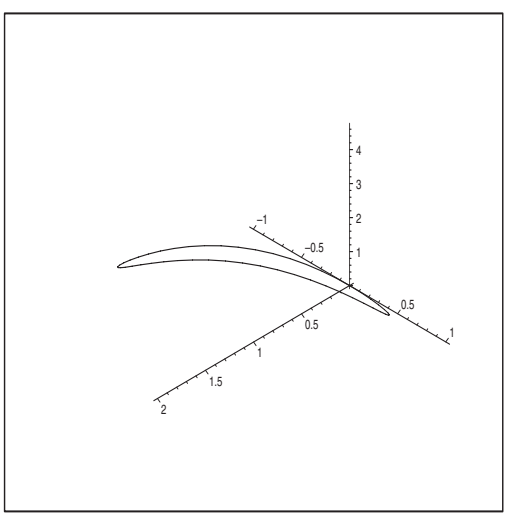

(a) $c_{1}(\theta)$

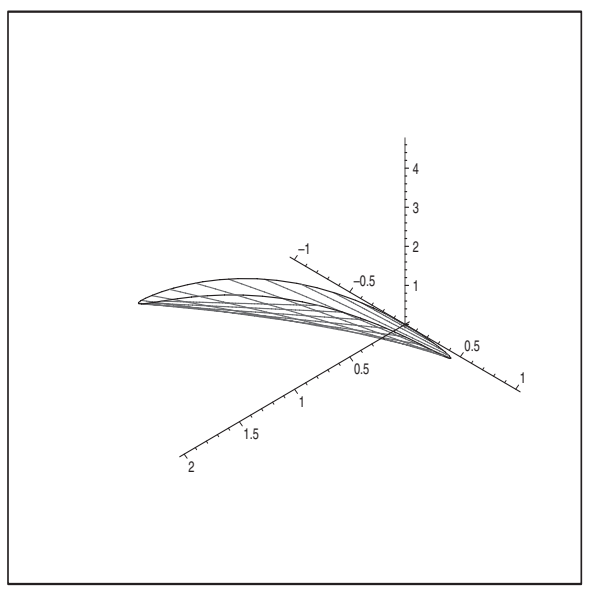

(c) Some rules of the spanning surface

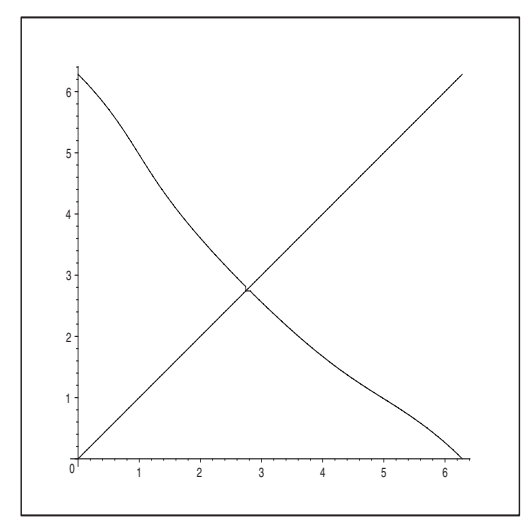

(b) $\theta$ vs. $\theta_{0}$

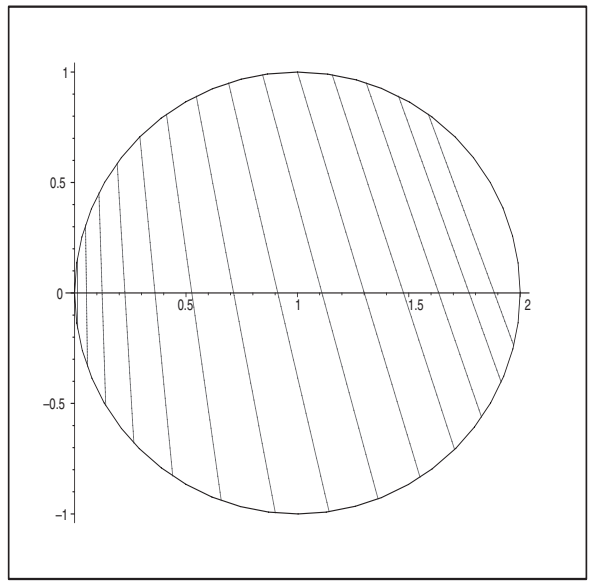

(d) Projection to the $x y$-plane

Figure 4. An example without an obstruction.

Of course, the example above is just about as well behaved as possible. However, the situation is often much more complicated. For example, if one considers the curve

$$
c(\theta)=(1-\cos (\theta), \sin (\theta), \sin (4 \sin (\theta)(1-\cos (\theta)))) .
$$


Figure 5 shows $\theta$ plotted as a function of $\theta_{0}$ (as in Figure $4 \mathrm{~b}$ in the previous example).

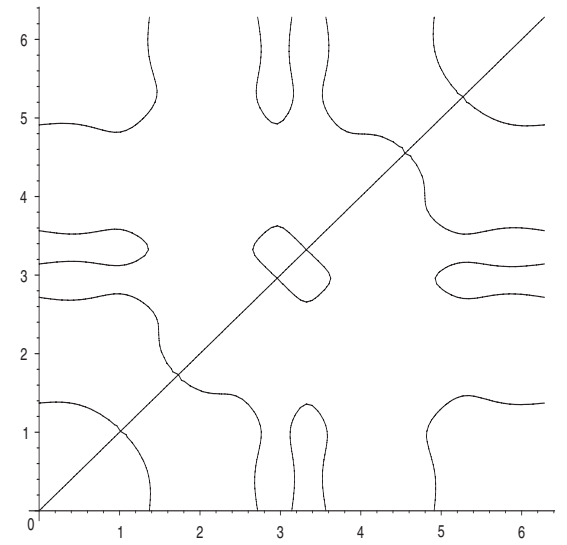

Figure 5. A more complicated example.

Example 3. In this example, we show an instance of the obstruction. In this case $\varphi^{\prime}(t)$ changes sign and we explore the effect of the sign change when building the ruled surface. Consider the curve (see Figure 6a)

$$
c_{2}(\theta)=\left(1-\cos (\theta), \sin (\theta), 1 / 5-1 / 5 \cos (\theta)+\sin ^{2}(\theta)\right) .
$$

In this case, as in the first case, $\theta_{0}=0$ is an isolated point and we can begin constructing a ruled surface. Figure $6 \mathrm{~b}$ shows $\theta$ plotted as a function of $\theta_{0}$. While tedious computation can confirm this, the figure clearly shows that $\varphi^{\prime}$ changes sign at roughly $\frac{\pi}{2}$. Figures $6 \mathrm{c}$ and $6 \mathrm{~d}$ illustrate how the construction fails - endpoints of the rules "backtrack" on the curve, creating a folded surface which, of course, is no longer a graph.

We end this discussion by noting the genericity of each of these classes. As the obstruction is defined by the monotonicity of $\varphi$, we note that strict monotonicity and non-monotonicity are open conditions in the $C^{1}$ topology by the implicit function theorem. To make this precise, we make the following definition.

Definition 6.6. A $C^{2}$ curve $c$ is generically nonmonotone if there exists an $\varepsilon>0$ so that for any associated function $\varphi$, there are parameter values $t_{1}, t_{2}$ so that $\varphi^{\prime}\left(t_{1}\right)>\varepsilon$ and $\varphi^{\prime}\left(t_{2}\right)<-\varepsilon$.

Proposition 6.7. Suppose for a given $c \in C^{2}$, there exists a $C^{1}$ ruled spanning $H$-minimal graph. If the associated function $\varphi$ is strictly monotone, then there ex- 
ists an open neighborhood, $G$, of $C^{2}$ closed curves with respect to the $C^{1}$ topology containing $c$ so that any curve in $G$ has no obstruction to building a $C^{1}$ ruled H-minimal spanning surface.

In addition, if $d$ is a $C^{2}$ generically nonmonotone curve, then there exists an open neighborhood of $d$ with respect to the $C^{1}$ topology so that any curve in this neighborhood cannot be spanned by a ruled minimal graph.

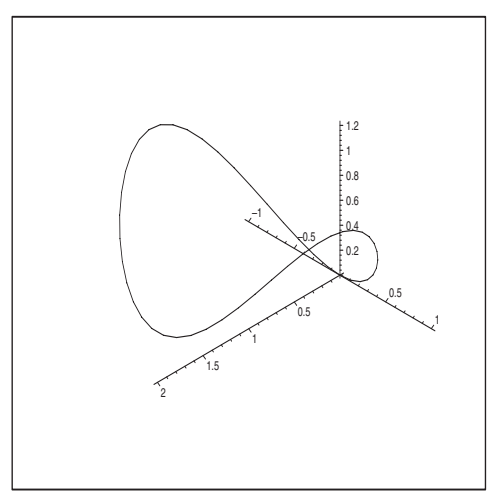

(a) $c_{2}(\theta)$

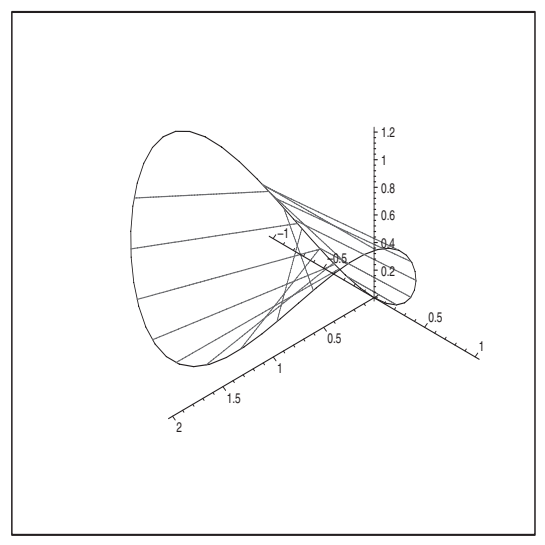

(c) Some rules of the spanning surface

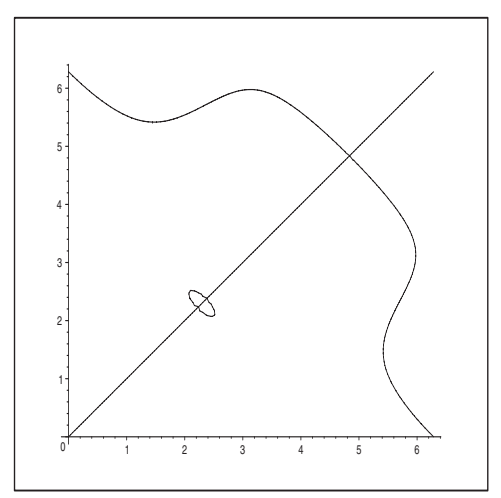

(b) $\theta$ vs. $\theta_{0}$

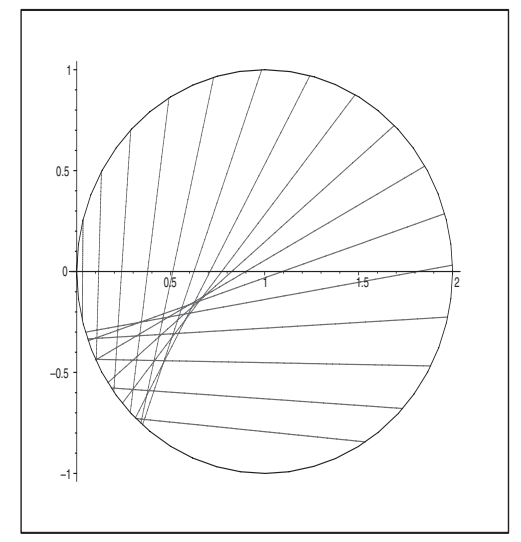

(d) Projection to the $x y$-plane

Figure 6. An example with an obstruction.

Proof. This is a consequence of formula (30). For example, assume that $\varphi$ is strictly 
monotone decreasing:

$$
\varphi^{\prime}(t)=\frac{c_{3}^{\prime}(t)+\frac{1}{2} c(\varphi(t)) \cdot c^{\prime}(t)^{\perp}}{c_{3}^{\prime}(\varphi(t))+\frac{1}{2} c(t) \cdot c^{\prime}(\varphi(t))^{\perp}}<-a^{2}<0 .
$$

Then, if we replace $c(t)$ with $\tilde{c}(t)=c(t)+\varepsilon(t)$ where $|\varepsilon(t)|<\delta$ and $\left|\varepsilon^{\prime}(t)\right|<\delta$, we have

$$
\begin{aligned}
\varphi_{\varepsilon}^{\prime}(t) & =\frac{\tilde{c}_{3}^{\prime}(t)+\frac{1}{2} \tilde{c}(\varphi(t)) \cdot \tilde{c}^{\prime}(t)^{\perp}}{\tilde{c}_{3}^{\prime}(\varphi(t))+\frac{1}{2} \tilde{c}(t) \cdot \tilde{c}^{\prime}(\varphi(t))^{\perp}} \\
& <-a^{2}+o_{\delta}(1) .
\end{aligned}
$$

Thus, for $\delta$ sufficiently small (i.e. $\tilde{c}$ sufficiently close to $c$ in the $C^{1}$ topology), $\varphi_{\varepsilon}^{\prime}$ is strictly monotone decreasing. A similar argument shows the same genericity result for curves where $\varphi$ is generically nonmonotone.

\subsection{Totally non-Legendrian curves}

Theorem 6.8. Suppose $c$ is a $C^{1}$ curve with no Legendrian points which is contained in an open $C^{1}$ ruled H-minimal graph, $S$. Then there exists an interval, I, so that $c(I)$ is contained in a plane.

Proof. We first record some easy observations:

- $S$ cannot have a characteristic point at any point of $c$. If $c\left(t_{0}\right)$ were a characteristic point, then any smooth curve through $c\left(t_{0}\right)$ would be tangent to $\mathscr{H}_{c\left(t_{0}\right)}$, including $c$ itself.

- Consider a point $c\left(t_{0}\right)$ and let $\gamma$ be a seed curve through $c\left(t_{0}\right)$. Use Theorem A to parameterize a neighborhood, $N$, of $c\left(t_{0}\right)$ that includes $c(t)$ for $t \in J$ where $J$ is an appropriate interval containing $t_{0}$. By the non-Legendrian assumption and continuity of the normal vector, we may assume that there are no characteristic points in $N$ and hence, by Theorem 3.8, $\gamma \in C^{2}$. Using the parametrization given by Theorem A, there exist functions $s(t)$ and $r(t)$ so that $c(t) \cap N$ is parametrized by

$$
\left(\gamma_{1}(s(t))+r(t) \gamma_{2}^{\prime}(s(t)), \gamma_{2}(s(t))-r(t) \gamma_{1}^{\prime}(s(t)), h_{0}(s(t))-\frac{r(t)}{2} \gamma \cdot \gamma^{\prime}(s(t))\right) .
$$

- A rule through $c\left(t_{0}\right)$ is transverse to $c(t)$. Indeed, if the rule were tangent then, by definition, $c\left(t_{0}\right)$ is Legendrian.

- For every $t \in J, \gamma(s(t))$ is twice differentiable and, applying formula (18) at these points determines the characteristic points along the rules passing through those points. 
Let $\mathcal{L}_{t}(r)$ be the rule through $c(t)$ and let $\overline{\mathcal{L}}_{t}(r)$ be the projection of the rule to the $x y$-plane.

Claim. There exists two rules of $S$ that intersect in the interior of the portion of $S$ bounded by $c$.

To show the claim, we assume there are no such rules. First pick a parameter value $\theta_{1}$ and let $\mathcal{L}_{\theta_{1}}(r)$ be the rule through $c\left(\theta_{1}\right)$. Under the assumption that the rule does not intersect any other rules inside $c$, it must intersect another point on $c$, dividing $c$ (and the surface) into two parts. Next, pick a parameter value, $\theta_{2}$, so that $c\left(\theta_{2}\right)$ is on the "left hand side" of the cut (see Figure 7). The rule, $\mathcal{L}_{\theta_{2}}(r)$ again must cut the remaining portion into two parts. We continue this iterative process, picking a sequence of parameter values $\left\{\theta_{i}\right\}$. By construction, this sequence must converge to a value $\theta_{\infty}$. Moreover, if the rule $\mathscr{L}_{\theta_{\infty}}(r)$ does not intersect any of the $\left\{\mathcal{L}_{\theta_{i}}(r)\right\}$, it must be tangent to $c$ at $\theta_{\infty}$. This implies that $c\left(\theta_{\infty}\right)$ is a Legendrian point, violating the hypothesis.

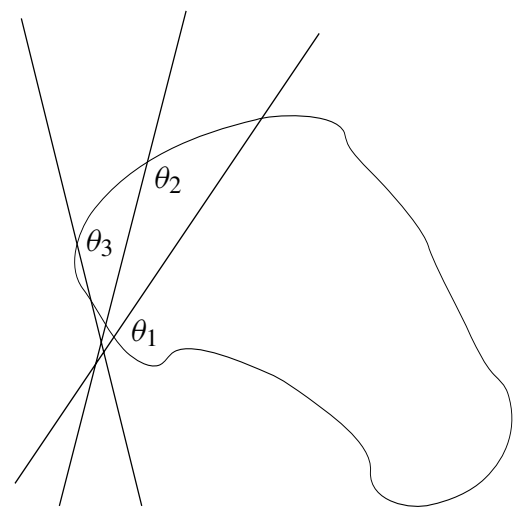

Figure 7. Heuristic for picking points in the proof of Theorem 6.8 .

Now, by the claim, we can pick $t_{1}, t_{2}$ so that $\overline{\mathcal{L}}_{t_{1}}(r) \cap \overline{\mathcal{L}}_{t_{2}}(r) \neq \emptyset$. Then the projection of these two rules must not be parallel and hence, as the projections are lines, they must intersect in a single point, $\{\bar{x}\}$. As $S$ is a graph over the $x y$-plane, we see that $\mathcal{L}_{t_{1}}(r) \cap \mathcal{L}_{t_{2}}(r)=\{x\}$ where $x$ is the point on $S$ over $\bar{x}$. By Lemma 3.11, $x$ must be a characteristic point of $S$. A consequence of this observation is that

$$
\bigcap_{t \in J} \mathcal{L}_{t}=\{x\}
$$

Suppose that this claim is not true, i.e. that there exists $t_{0}$ so that $\mathscr{L}_{t_{1}}(r) \cap \mathcal{L}_{t_{0}}(r)=$ $\left\{x^{\prime}\right\} \neq\{x\}$. Then along $\mathscr{L}_{t_{1}}$ there must be 2 characteristic points. By Lemma 3.12, 
as $S$ is a graph over the $x y$-plane, this cannot happen. Using a left translation, we may assume that $\{x\}$ is the origin. Let $I$ be the interval between $t_{0}$ and $t_{1}$.

Denote the portion of $S$ bounded by $c(I), \mathcal{L}_{t_{0}}(r)$ and $\mathcal{L}_{t_{1}}(r)$ by $S_{0}$. We finish the proof by showing that $S_{0}$ is a portion of a plane. Since the origin is a characteristic point, $S$ must be tangent to the $x y$-plane at the origin. As each rule is a horizontal straight line and every rule in $S_{0}$ passes through the origin, we have that every such rule lies in the $x y$-plane. Thus, $S_{0}$ is a piece of the $x y$-plane and so $c(I)$ is planar.

Corollary 6.9. If $c$ is a smooth curve with no Legendrian points and no portion of $c$ is contained in a plane then c cannot be spanned by a $C^{1}$ ruled $\mathrm{H}$-minimal graph.

In particular, the solution to the Plateau Problem for such a curve cannot be $C^{2}$ and, if it is $C^{1}$, cannot be a ruled surface. The best result in this case would be a $C^{1}$ $H$-minimal surface composed of ruled $C^{1} H$-minimal patches glued along common intersections.

Example 4. Let

$$
c(\theta)=(1-\cos (\theta), \sin (\theta), f(\theta)) .
$$

Then,

$$
c^{\prime}(\theta)=\sin (\theta) X_{1}+\cos (\theta) X_{2}+\left(f^{\prime}(\theta)-\frac{\cos (\theta)}{2}+\frac{1}{2}\right) T .
$$

Thus, for any periodic $f$ so that

$$
\left|f^{\prime}(\theta)-\frac{\cos (\theta)}{2}+\frac{1}{2}\right|>\varepsilon>0
$$

for some fixed $\varepsilon$, we have an example that is totally non-Legendrian.

An explicit example is given by

$$
f(\theta)=\frac{1}{2} \sin (\theta)+\frac{1}{8} \sin ^{2}(\theta)
$$

It is easy to show that no portion of this curve is planar.

\section{A. Integral curves of continuous vector fields}

In this appendix, we will review the existence of integral curves for continuous vector fields and prove some results needed in the main body of the paper. The results here are consequences of Picard's standard iterative construction of solutions to first order ordinary differential equations (see, for example, [13]). Our only modification is to restrict our view to merely continuous vector fields (as opposed to Lipschitz continuous ones). We make the following standing assumptions: 
(1) Let $X$ be a vector field defined on a compact domain $\Omega$.

(2) Let $\left\{X_{k}\right\}$ be a sequence of $C^{\infty}$ vector fields, defined on $\Omega$, which converge uniformly to $X$ on $\Omega$.

Let $\left\{M_{k}\right\}$ be a sequence constants tending to zero so that $\left|X_{k}-X\right| \leq M_{k}$ on $\Omega$. By compactness and the continuity of $X$, there exist constant $M$ and a non-increasing continuous function $C: \mathbb{R}_{+} \rightarrow \mathbb{R}_{+}$with $C(0)=0$ so that

$$
M=\max _{\Omega}|X|
$$

and for $x, y \in \Omega$,

$$
|X(x)-X(y)| \leq C(|x-y|) .
$$

By the compactness of $\Omega$ and the continuity of $X_{k}$, we have, for each $k$, constants $M(k)$ and non-increasing continuous functions $C_{k}: \mathbb{R}_{+} \rightarrow \mathbb{R}_{+}$with $C_{k}(0)=0$ so that

$$
M(k)=\max _{\Omega}\left|X_{k}\right| .
$$

Moreover, since $X_{k} \rightarrow X$ uniformly on $\Omega$, there exists a constant $\alpha$ so that

$$
M(k) \leq \alpha M
$$

for all $k$. Next, we construct integral curves for $X$ and $X_{k}$ emanating from a point $x_{0} \in \Omega$ using Picard's approximation method. To do so, let

$$
\varphi_{0}^{0}(t)=0, \quad \varphi_{0}^{k}(t)=0
$$

and

$$
\varphi_{n}^{0}(t)=x_{0}+\int_{0}^{t} X\left(\varphi_{n-1}^{0}(s)\right) d s, \quad \varphi_{n}^{k}(t)=x_{0}+\int_{0}^{t} X_{k}\left(\varphi_{n-1}^{k}(s)\right) d s .
$$

Lemma A.1. $\left\{\varphi_{n}^{0}\right\}$ has a subsequence which converges uniformly on $\Omega$.

Proof. First, since

$$
\left|\varphi_{n}^{0}(t)\right|=\left|x_{0}+\int_{0}^{t} X\left(\varphi_{n-1}^{0}(s)\right) d s\right| \leq\left|x_{0}\right|+\int_{0}^{t}\left|X\left(\varphi_{n-1}^{0}(s)\right)\right| d s \leq\left|x_{0}\right|+M t
$$

we have that the sequence is pointwise bounded. Second, since

$$
\left|\varphi_{n}^{0}\left(t_{1}\right)-\varphi_{n}^{0}\left(t_{0}\right)\right|=\left|\int_{t_{0}}^{t_{1}} X\left(\varphi_{n-1}(s)\right) d s\right| \leq M\left|t_{1}-t_{0}\right|
$$

we have that the sequence is equicontinuous (in fact uniformly Lipschitz). By the theorem of Arzela-Ascoli, there exists a subsequence that converges uniformly on $\Omega$. 
We note that the same argument applies (with appropriately defined constants) for $\left\{\varphi_{n}^{k}\right\}$ as well:

Lemma A.2. $\left\{\varphi_{n}^{k}\right\}_{k, n}$ has a subsequence which converges uniformly (in both $k$ and $n$ ) on $\Omega$.

Proof. As in the previous lemma, we have

$$
\left|\varphi_{n}^{k}(t)\right| \leq\left|x_{0}\right|+\int_{0}^{t}\left|X_{k}\left(\varphi_{n-1}^{k}(s)\right)\right| d s \leq\left|x_{0}\right|+t M(k) \leq\left|x_{0}\right|+t \alpha M .
$$

In other words, the sequence is pointwise bounded in both $k$ and $n$. Next,

$$
\left|\varphi_{n}^{k}\left(t_{1}\right)-\varphi_{n}^{k}\left(t_{0}\right)\right| \leq \int_{t_{0}}^{t_{1}}\left|X_{k}\left(\varphi_{n-1}^{k}(s)\right)\right| d s \leq M(k)\left|t_{1}-t_{0}\right| \leq \alpha M\left|t_{1}-t_{0}\right| .
$$

And so the sequence is equicontinuous as well. Thus, by the theorem of ArzelaAscoli, we may extract a subsequence that converges uniformly in both $k$ and $n$ on $\Omega$.

For the purposes of this discussion, we assume that we have taken the appropriate subsequences so that $\varphi_{n}^{0} \rightarrow \varphi^{0}$ and $\varphi_{n}^{k} \rightarrow \varphi^{k}$ uniformly on $\Omega$. This gives us the existence of integral curves for these vector fields. Of course, these integral curves may not be unique.

We next show that, using these integral curves, $X_{k}\left(\varphi^{k}(t)\right) \rightarrow X\left(\varphi^{0}(t)\right)$.

\section{Lemma A.3.}

$$
\left|\varphi_{n}^{k}(t)-\varphi_{n}^{0}(t)\right| \leq M_{k} t+C^{n-1}(k, t)
$$

where

$$
C^{m}(k, t)=t C\left(M_{k} t+t C\left(M_{k} t+t C\left(M_{k} t+\cdots+t C\left(M_{k} t\right)\right)\right)\right)
$$

and the nested applications of the function $C$ occur $m$ times.

Proof. We proceed by induction. First, we note several initial cases:

$$
\begin{gathered}
\left|\varphi_{0}^{k}(t)-\varphi_{0}^{0}(t)\right|=0, \\
\left|\varphi_{1}^{k}(t)-\varphi_{1}^{0}(t)\right| \leq \int_{0}^{t}\left|X_{k}(0)-X(0)\right| d s \leq M_{k} t=M_{k} t+C^{0}(k, t),
\end{gathered}
$$




$$
\begin{aligned}
\left|\varphi_{2}^{k}(t)-\varphi_{2}^{0}(t)\right| & \leq \int_{0}^{t}\left|X_{k}\left(\varphi_{1}^{k}(s)\right)-X\left(\varphi_{1}^{0}(s)\right)\right| d s \\
& \leq M_{k} t+\int_{0}^{t}\left|X\left(\varphi_{1}^{k}(s)\right)-X\left(\varphi_{1}^{0}(s)\right)\right| d s \\
& \leq M_{k} t+\int_{0}^{t} C\left(\left|\varphi_{1}^{k}(s)-\varphi_{1}^{0}(s)\right|\right) d s \\
& \leq M_{k} t+t C\left(M_{k} t\right) \quad \text { (by the previous calculation) } \\
& =M_{k} t+C^{1}(k, t) .
\end{aligned}
$$

Now assume that

$$
\left|\varphi_{n-1}^{k}(t)-\varphi_{n-1}^{0}(t)\right| \leq M_{k} t+t C^{n-2}(k, t) .
$$

Then

$$
\begin{aligned}
\left|\varphi_{n}^{k}(t)-\varphi_{n}^{0}(t)\right| & \leq \int_{0}^{t}\left|X_{k}\left(\varphi_{n-1}^{k}(s)\right)-X\left(\varphi_{n-1}^{0}(s)\right)\right| d s \\
& \leq M_{k} t+\int_{0}^{t}\left|X\left(\varphi_{n-1}^{k}(s)\right)-X\left(\varphi_{n-1}^{0}(s)\right)\right| d s \\
& \leq M_{k} t+\int_{0}^{t} C\left(\left|\varphi_{n-1}^{k}(s)-\varphi_{n-1}^{0}(s)\right|\right) d s \\
& \leq M_{k} t+\int_{0}^{t} C\left(M_{k} t+C^{n-1}(k, t)\right) d s \quad \text { (by the induction hypothesis) } \\
& \leq M_{k} t+t C\left(\left(M_{k} t+C^{n-1}(k, t)\right)\right)=M_{k} t+C^{n}(k, t) .
\end{aligned}
$$

This completes the induction and the proof.

We note that as $M_{k}$ is a coefficient in every term of each argument of $C$ in $C^{m}(k, t)$ and $C(0)=0$, we have that $\lim _{k \rightarrow \infty} C^{m}(k, t)=0$ as $\lim _{k \rightarrow \infty} M_{k}=0$. Moreover, in light of Lemma A.2, we know that $\varphi_{n}^{k}$ tends to some function uniformly as $k \rightarrow \infty$, we see that the previous lemma implies that $\varphi_{n}^{k} \rightarrow \varphi_{n}^{0}$ as $k \rightarrow \infty$.

We now prove the claim:

Lemma A.4. $\lim _{k \rightarrow \infty}\left|X_{k}\left(\varphi^{k}(t)\right)-X\left(\varphi^{0}(t)\right)\right|=0$.

Proof.

$$
\begin{aligned}
\lim _{k \rightarrow \infty}\left|X_{k}\left(\varphi^{k}(t)\right)-X\left(\varphi^{0}(t)\right)\right| & =\lim _{k \rightarrow \infty} \lim _{n \rightarrow \infty}\left|X_{k}\left(\varphi_{n}^{k}(t)\right)-X\left(\varphi_{n}^{0}(t)\right)\right| \\
& =\lim _{n \rightarrow \infty} \lim _{k \rightarrow \infty}\left|X_{k}\left(\varphi_{n}^{k}(t)\right)-X\left(\varphi_{n}^{0}(t)\right)\right| \\
& =\lim _{n \rightarrow \infty}\left|X\left(\varphi_{n}^{0}(t)\right)-X\left(\varphi_{n}^{0}(t)\right)\right|=0 .
\end{aligned}
$$


In the second equality, we may switch the order of the limits because $\varphi_{n}^{k} \rightarrow \varphi^{k}$ uniformly in both $\Omega$ and $k$ as $n \rightarrow 0$ by Lemma A.2.

\section{References}

[1] M. Bonk and L. Capogna, Mean curvature flow in the Heisenberg group. Preprint, 2005.

[2] L. Capogna, D. Danielli, and N. Garofalo, The geometric Sobolev embedding for vector fields and the isoperimetric inequality. Comm. Anal. Geom. 2 (1994), 203-215. Zbl 0864.46018 MR 1312686

[3] L. Capogna, D. Danielli, and N. Garofalo, An isoperimetric inequality and the geometric Sobolev embedding for vector fields. Math. Res. Lett. 1 (1994), 263-268. Zbl 0861.46019 MR 1266765

[4] J.-H. Cheng and J.-F. Hwang, Properly embedded and immersed minimal surfaces in the Heisenberg group. Preprint, arxiv math.DG/0407094, 2004.

[5] J.-H. Cheng, J.-F. Hwang, A. Malchiodi, and P. Yang, Minimal surfaces in pseudohermitian geometry. Preprint, arxiv math.DG/0401136, 2003.

[6] D. Cole, On minimal surfaces in Martinet-type spaces. PhD thesis, Dartmouth College, 2005.

[7] D. Danielli, N. Garofalo, and D.-M. Nhieu, Minimal surfaces, surfaces of constant mean curvature and isoperimetry in Carnot groups. Preprint, August, 2001.

[8] H. Federer, Geometric measure theory. Grundlehren Math. Wiss. 153, Springer-Verlag New York Inc., New York 1969. Zbl 0176.00801 MR 0257325

[9] B. Franchi, S. Gallot, and R. L. Wheeden, Sobolev and isoperimetric inequalities for degenerate metrics. Math. Ann. 300 (1994), 557-571. Zbl 0830.46027 MR 1314734

[10] B. Franchi, R. Serapioni, and F. Serra Cassano, Rectifiability and perimeter in the Heisenberg group. Math. Ann. 321 (2001), 479-531. Zbl 1057.49032 MR 1871966

[11] N. Garofalo and D.-M. Nhieu, Isoperimetric and Sobolev inequalities for Carnot-Carathéodory spaces and the existence of minimal surfaces. Comm. Pure Appl. Math. 49 (1996), 1081-1144. Zbl 0880.35032 MR 1404326

[12] N. Garofalo and S. D. Pauls, The Bernstein problem in the Heisenberg group. Submitted, arxiv math.DG/0209065, 2003.

[13] P. Hartman, Ordinary differential equations. Second edition, Birkhäuser, Boston, MA, 1982. Zbl 0476.34002 MR 0658490

[14] R. Hladky and S. D. Pauls, Minimal surfaces in the Roto-translation group. Preprint, arxiv math.DG/0509636, 2005.

[15] R. K. Hladky and S. D. Pauls, Constant mean curvature surfaces in sub-riemannian spaces. Submitted, arxiv math.DG/0508333, 2005.

[16] S. D. Pauls, Minimal surfaces in the Heisenberg group. Geom. Dedicata 104 (2004), 201-231. Zbl 1054.49029 MR 2043961

[17] M. Ritoré and C. Rosales, Rotationally invariant hypersurfaces with constant mean curvature in the Heisenberg group $\mathbb{H}^{n}$. Preprint, arxiv math.DG/0504439, 2005. 
Received January 25, 2005

Scott D. Pauls, Dartmouth College, Hanover, NH 03755, U.S.A.

E-mail: scott.pauls@dartmouth.edu 